\title{
Pathways for diffusion in the potential energy landscape of the network glass former $\mathrm{SiO}_{2}$
}

S. P. Niblett, M. Biedermann, D. J. Wales, and V. K. de Souza

Citation: The Journal of Chemical Physics 147, 152726 (2017);

View online: https://doi.org/10.1063/1.5005924

View Table of Contents: http://aip.scitation.org/toc/jcp/147/15

Published by the American Institute of Physics

\section{Articles you may be interested in}

Defining and quantifying frustration in the energy landscape: Applications to atomic and molecular clusters, biomolecules, jammed and glassy systems

The Journal of Chemical Physics 146, 124103 (2017); 10.1063/1.4977794

Preface: Special Topic on Reaction Pathways

The Journal of Chemical Physics 147, 152401 (2017); 10.1063/1.5007080

Perspective: Highly stable vapor-deposited glasses

The Journal of Chemical Physics 147, 210901 (2017); 10.1063/1.5006265

Predicting reaction coordinates in energy landscapes with diffusion anisotropy

The Journal of Chemical Physics 147, 152701 (2017); 10.1063/1.4983727

Transition path sampling of rare events by shooting from the top

The Journal of Chemical Physics 147, 152716 (2017); 10.1063/1.4997378

Perspective: Surface freezing in water: A nexus of experiments and simulations

The Journal of Chemical Physics 147, 060901 (2017); 10.1063/1.4985879

\section{AIP | The Jounal of AIP $\left.\right|_{\text {Chemical Phyics }}$} PERSPECTIVES 


\title{
Pathways for diffusion in the potential energy landscape of the network glass former $\mathrm{SiO}_{2}$
}

\author{
S. P. Niblett, ${ }^{1, a)}$ M. Biedermann, ${ }^{2, b)}$ D. J. Wales, ${ }^{1, c)}$ and V. K. de Souza ${ }^{1, d)}$ \\ ${ }^{1}$ University Chemical Laboratories, Lensfield Road, Cambridge CB2 1EW, United Kingdom \\ ${ }^{2}$ Institute of Physical Chemistry, University of Muenster, Corrensstraße 28/30, 48149 Muenster, Germany
}

(Received 8 May 2017; accepted 20 September 2017; published online 16 October 2017)

\begin{abstract}
We study the dynamical behaviour of a computer model for viscous silica, the archetypal strong glass former, and compare its diffusion mechanism with earlier studies of a fragile binary LennardJones liquid. Three different methods of analysis are employed. First, the temperature and time scale dependence of the diffusion constant is analysed. Negative correlation of particle displacements influences transport properties in silica as well as in fragile liquids. We suggest that the difference between Arrhenius and super-Arrhenius diffusive behaviour results from competition between the correlation time scale and the caging time scale. Second, we analyse the dynamics using a geometrical definition of cage-breaking transitions that was proposed previously for fragile glass formers. We find that this definition accurately captures the bond rearrangement mechanisms that control transport in open network liquids, and reproduces the diffusion constants accurately at low temperatures. As the same method is applicable to both strong and fragile glass formers, we can compare correlation time scales in these two types of systems. We compare the time spent in chains of correlated cage breaks with the characteristic caging time and find that correlations in the fragile binary Lennard-Jones system persist for an order of magnitude longer than those in the strong silica system. We investigate the origin of the correlation behaviour by sampling the potential energy landscape for silica and comparing it with the binary Lennard-Jones model. We find no qualitative difference between the landscapes, but several metrics suggest that the landscape of the fragile liquid is rougher and more frustrated. Metabasins in silica are smaller than those in binary Lennard-Jones and contain fewer high-barrier processes. This difference probably leads to the observed separation of correlation and caging time scales. Published by AIP Publishing. https://doi.org/10.1063/1.5005924
\end{abstract}

\section{INTRODUCTION}

Glasses are mechanical solids that lack the long-range order of a crystalline structure. They can be formed by cooling a viscous liquid fast enough that crystallisation is avoided, which is known as supercooling. Below the melting temperature of the crystal, if the liquid does not crystallise, it exists in a metastable state as a supercooled liquid. On further cooling, viscosity increases, relaxation times increase, and self-diffusion slows down. Eventually, when the structure appears frozen on an experimental time scale, the system can be described as a glass.

However, not all supercooled liquids behave in the same way as they are cooled down to the glass transition. For some supercooled liquids, the temperature dependence of relaxation times or transport properties is stronger than the Arrhenius law. These liquids display "super-Arrhenius" behaviour, which can be interpreted as resulting from an increasing barrier height with falling temperature. The Vogel-TammannFulcher (VTF) equation ${ }^{1-3}$ is commonly used to fit such behaviour.

\footnotetext{
a)sn402@cam.ac.uk

b)m.biedermann@wwu.de

c)dw34@cam.ac.uk

d)vkd21@cam.ac.uk
}

Angell classified supercooled liquids as "strong" or "fragile" by their degree of super-Arrhenius behaviour. ${ }^{4-6}$ Fragility can be quantified using thermodynamic or kinetic definitions. In fragile liquids, the glass transition is often associated with a significant peak in the heat capacity at $T_{g}$, while strong liquids exhibit smoother changes in dynamical properties. Thermodynamic and kinetic fragilities often correlate very well, which may be evidence of a common cause at the atomic level.

Structural signatures can also be a measure of fragility. ${ }^{7,8}$ "Strong" materials include many network glass formers, such as silica and germanium dioxide, which have tetrahedrally coordinated structures, while "fragile" materials, such as ortho-terphenyl, are often bound by dispersive and less directional forces. It is likely that the structural differences in strong and fragile materials lead to different diffusion mechanisms. Understanding these mechanisms and rates would be a key step towards explaining the differences between strong and fragile supercooled liquids.

In this paper, we provide a simplified description of the diffusion mechanism for silica, the archetypal strong glass former, and examine the differences between strong and fragile systems.

\section{A. Diffusion}

For supercooled liquids, the mean square displacement used to calculate diffusion constants is usually shown on 
a double logarithmic plot, which facilitates examination of dynamics on various time scales. At short times, there is a power-law behaviour with an exponent of two, which corresponds to the initial ballistic motion of the particles. At long times, the behaviour can again be described by a power law, but with an exponent of one, indicating diffusive behaviour. In the high-temperature limit, these two regimes are directly connected, but in the low-temperature limit, the particles can appear almost frozen, as a plateau region develops between the two extremes. This plateau region is generally associated with trapping within a cage of neighbouring particles, ${ }^{9}$ from which escape is relatively slow. The mechanism for long-time diffusion involves successive escapes from nearestneighbour cages. As we approach the glass transition, cage escapes become rare events. Both fragile ${ }^{9}$ and strong ${ }^{10}$ supercooled liquids exhibit this plateau region in the mean-square displacement.

\section{Short-time diffusion constants}

The effect of the observation time scale on the apparent diffusion constants has been investigated previously for two model fragile glass formers: binary Lennard-Jones ${ }^{11,12}$ (BLJ) and ortho-terphenyl $^{13}$ (OTP). Long (locally ergodic) ${ }^{14}$ molecular dynamics trajectories were divided into a series of short non-ergodic intervals of length $\tau$. The reduced diffusion constant $D(\tau)$ was then calculated using a mean squared displacement evaluated over each interval of length $\tau$. This method neglects any correlation that may be present between different time intervals. For fragile liquids, $D(\tau)$ shows an Arrhenius temperature dependence for small values of $\tau$. For large $\tau$, as the interval length approaches local ergodicity and $D(\tau)$ tends towards the values for the full trajectory, super-Arrhenius curvature reappears. An approximate correlation term, based on the average angle between displacements in successive time windows, recovers the full super-Arrhenius behaviour. ${ }^{11,13}$ These results show that super-Arrhenius diffusion results from a quantifiable correlation effect. Displacements in a given time window often directly reverse displacements in previous windows. ${ }^{11}$ This method is particularly useful for characterising reversals in very large or complicated systems, where cage breaks are difficult to identify. ${ }^{13}$

In Sec. III we will study short-time diffusion constants and reversals for silica in detail.

\section{The cage effect}

We can examine the effect of reversals in more detail by identifying processes where atoms break out of their nearestneighbour cages. The cage effect has been used in several different ways to probe the dynamics of supercooled liquids. In simulations ${ }^{15,16}$ and experiments, ${ }^{17,18}$ three-time correlation functions show that the dynamics at short times are dominated by the presence of the cage, which results in a systematic backdragging effect. Caging is also an important concept in the mode-coupling theory (MCT) of supercooled dynamics. ${ }^{19,20}$ Dynamics in the deeply supercooled regime can be separated into non-diffusive "cage-rattling motions" and diffusive "cage-breaking" rearrangements. . $^{21,22}$

Rabani et al. developed a "cage correlation function" based on changes in the nearest neighbours for a particular atom, to estimate the typical residence time within a particular configuration of cages ${ }^{23,24}$ and reproduce the non-exponential relaxation behaviour of fragile glass formers. ${ }^{25,26}$

It is possible to identify rearrangements that involve transitions of atoms between cages. ${ }^{22,27-31}$ It has been observed that cage-changing motions are often rapidly reversed, and the proportion of reversals increases with decreasing temperature. ${ }^{27-30,32}$ By examining local minima on the potential energy landscape, rather than configurations from a trajectory, de Souza and Wales proposed a microscopic definition of a local cage-breaking process involving changes in nearest neighbours. ${ }^{13,30}$ The diffusion constant can be approximated using only atomic displacements resulting from productive (non-reversed) cage-breaking transitions, indicating that these transitions are the most important transport processes involved in long-time diffusion.

For fragile supercooled liquids, it has been shown that diffusion can be described as a correlated random walk: the steps are cage breaks, and a correlation factor accounts for direct return events. ${ }^{33}$ A continuous-time random walk has also been used to study the dynamics in glass formers. ${ }^{34,35}$ A random walk is a natural description for diffusion in strong network glass formers like silica, either in the context of bondbreaking ${ }^{10,36}$ or cage breaking. ${ }^{35}$ The question we wish to answer in this paper concerns the correlation aspect of such a random walk. In Sec. IV, we apply a cage-breaking analysis to silica and examine cage-breaking reversals for this system.

\section{B. Potential energy landscapes}

A potential energy surface or landscape (PEL) represents the potential energy of a given system as a function of all the relevant atomic or molecular coordinates. A connection between the properties of glasses and the potential energy surface was originally proposed by Goldstein. ${ }^{37}$ Goldstein proposed the involvement of two distinct time scales: fast motion involving vibrations about local minima of the PEL and less frequent jumps over significant energy barriers separating different minima.

Generally, as the temperature is lowered, the influence of the potential energy landscape is felt more strongly. For the fragile BLJ mixture at number density 1.2, potential energy barriers appear to have a significant influence on dynamics below a temperature of 0.9 reduced units. ${ }^{38}$ This temperature is approximately $2 T_{C}$, where $T_{C}$ is the mode coupling critical temperature obtained by fitting diffusion data. ${ }^{9,39}$ Below $T=0.9$, the system is said to enter a "landscape-influenced" regime, ${ }^{38}$ across which the average potential energy of the minima decreases. On further cooling, a low-temperature plateau in the energy of the minima begins, close to the predicted $T_{C}$, forming a "landscape-dominated regime," where the average energy of local minima sampled varies with cooling rate. ${ }^{38,40}$ For silica, our archetypal strong glass former, local minimum energies behave in a similar manner. ${ }^{41,42}$ The average energy of the minima starts to fall at $T \approx 10000 \mathrm{~K}$ and a low-temperature plateau appears below $T \approx 3500 \mathrm{~K}$, close to the predicted $T_{C}=3300 \mathrm{~K} .{ }^{10}$ Jund and Jullien showed ${ }^{43}$ that below $3300 \mathrm{~K}$ the mean square distance between an instantaneous configuration visited by a molecular dynamics (MD) trajectory and the corresponding local minimum depends 
linearly on the temperature, but above this intrinsic temperature, the mean square distance increases much more rapidly. A similar trend was observed in the energy difference between the instantaneous configurations and corresponding local minima.

For several different glass formers a wide range of barriers was found, from high barriers corresponding to several pair well depths, where one or more atoms change their nearestneighbour coordination shell, down to processes with very small activation energies, where all nearest-neighbour cages are preserved ${ }^{44,45}$ Kushima et al. studied transition states in silica located by a basin-filling procedure and found a similar separation of rearrangement mechanisms into high- and low-barrier transitions. ${ }^{41}$ The high-barrier processes corresponded to an $\mathrm{Si}-\mathrm{O}$ bond breaking and immediately reforming, while the low-barrier processes involved reaction of an undercoordinated oxygen atom (a "dangling bond") with an undercoordinated silicon atom.

We explore the potential energy landscape for silica in Sec. V, visualising the landscape and identifying bondbreaking transitions as cage breaks.

\section{Metabasins}

A metabasin ${ }^{13,30,46,47}$ may be defined as a set of minima that the system can traverse rapidly and reversibly. ${ }^{48-51}$ Metabasin transitions are much less frequent than intrametabasin transitions and are effectively irreversible, thus reducing diffusion to a random walk between metabasins.

Metabasins can be defined using an algorithm that considers revisits to minima previously visited in the course of the trajectory. ${ }^{50,52}$ An identical coarse-grained description can be provided by a cage-breaking analysis and associating productive cage breaks with transitions between metabasins. ${ }^{13,30}$ In this description, both intra-cage motion and reversed cage-breaking transitions take place within a metabasin.

Saksaengwijit and Heuer ${ }^{33}$ performed a detailed analysis of the structure of metabasins for a silica system of 99 particles with periodic boundary conditions, attributing the negative correlation of motion within a metabasin to entropic effects favouring reversals of minimum-minimum transitions. In Sec. V C, we identify metabasins for a larger system of 555 particles. Additionally we visualise these metabasins in the landscape and compare them to those found for fragile glass formers.

\section{Comparing strong and fragile glass formers}

Numerous attempts have been made to explain the differences between strong and fragile glass formers. However, this subject is clouded by a number of factors. For a particular material, fragility can be changed by factors such as density $^{5,54}$ and pressure. ${ }^{55,56}$ Even for silica, the archetypal strong material, there is a "fragile-strong crossover" $10,57-59$ in the diffusion constant, above which the apparent energy barrier increases with increasing temperature. This deviation from straight-line Arrhenius behaviour occurs at $3221 \mathrm{~K}$ in experimental measurements of viscosity ${ }^{57,58}$ and around 3300$3500 \mathrm{~K}$ in simulations. ${ }^{53,59,60}$ Some authors ${ }^{59}$ associate the crossover with a feature in the heat capacity. Others ${ }^{10}$ argue that these diffusion constants are best fitted by a power law in $\left(T-T_{c}\right)$, where $T_{c}$ is the mode-coupling critical temperature, and hence the crossover is associated with the transition from flow-like to hopping particle motion that is predicted by mode coupling theory. If the high-temperature behaviour can indeed be explained by mode coupling theory, then it arises from a different mechanism to the low-temperature superArrhenius behaviour of fragile glass formers, and the term "fragile-strong" is misleading. Another possibility ${ }^{61}$ is that the high-temperature behaviour is not super-Arrhenius at all, and the crossover simply connects two different Arrhenius regimes.

The Arrhenius behaviour of strong materials is often viewed as the simple case of supercooled transport behaviour, corresponding to thermal activation over a fixed energy barrier by local, noncooperative rearrangement events. However, it has recently been suggested that strong and fragile materials are not different classes of materials, but rather that network liquids should be considered as an extreme case in the general class of fragile systems. ${ }^{61}$ Many signatures of fragile systems are also present in strong systems, but have different character or are present to a weaker degree. These signatures include spatially heterogeneous dynamics and correlated motion of particles in string-like paths ${ }^{61-64}$ and also correlated motion in the form of reversals. ${ }^{42}$ A continuous-time random walk can be used to describe the dynamics of both silica and a fragile polymer melt, suggesting that dynamics is universal at the level of single-particle jumps. ${ }^{35}$

In terms of the potential energy landscape, the simplest view would suggest that the organisation and connectivity of the landscape is qualitatively different for strong materials. It has been suggested that the division of a glassy landscape into metabasins may be sufficient to produce super-Arrhenius behaviour. ${ }^{46}$ However, as metabasins and negative correlations are still present for strong systems, the difference may well be more subtle. Saksaengwijit et al. ${ }^{34,36,42,53}$ have found many similarities in the ways strong and fragile supercooled liquids explore the PEL, but also some key differences. For small system sizes of silica, the influence of a low-energy cutoff in the distribution of potential energy minima is seen at temperatures below $4000 \mathrm{~K} .^{42}$ This cutoff is thought to correspond to the limit of zero defects in the coordination number. It is argued that the existence of this cutoff is sufficient to explain the fragile-strong crossover in the diffusion constant ${ }^{10,42,53}$ and combined with a narrow distribution of metabasin escape barriers leads to Arrhenius behaviour ${ }^{53}$ as the effective energy barrier to diffusion does not increase.

Kushima $\mathrm{et} \mathrm{al.}{ }^{41}$ coarse-grained the energy landscape by determining a single effective activation barrier to diffusion as a function of temperature. They suggest that at very high and very low temperatures, this barrier becomes effectively constant, leading to strong behaviour, but a fragile regime exists in between these where the barrier changes as a function of temperature. For a strong liquid, the transition between barriers is fast, so the "fragility zone" is narrow and most experiments and simulations will only detect the strong regime. For fragile liquids, the transition zone between the plateaux covers most of the accessible temperature range. The key prediction of this theory is for two fragile-strong crossovers to exist for every supercooled liquid. 
Previously, we have shown that the super-Arrhenius behaviour of both the BLJ fluid and OTP arise from a quantifiable negative correlation in particle motion, which increases at lower temperatures. ${ }^{11-13}$ These negative correlations were interpreted in terms of a microscopic definition for cagebreaking processes ${ }^{13,30}$ and the connectivity of the potential energy landscape including metabasins. ${ }^{33}$

In this paper, we study silica, a strong network glass former. If strong glass formers are an extreme case of fragile systems, we would expect that negative correlation is still present but less important than for fragile liquids. This hypothesis is tested in Sec. III. The network character of silica lends itself naturally to a description of diffusion involving cage breaking. In Sec. IV we will show that our definition of cagebreaking events is equally applicable to silica and BLJ, which allows direct comparison of diffusion mechanisms between the two systems. We also know that metabasins exist for silica, but again we expect to identify differences to those found for fragile systems. In Sec. V we explore the PEL of silica directly and show that cage breaks are intrinsically linked to the structure of the PEL, but that geometrical metabasins are smaller and less relevant than for fragile liquids. Finally, Sec. VI contains some simple efforts to compare the energy landscapes of silica and BLJ, exploring further the origins of the differences between strong and fragile glass formers.

\section{TECHNICAL DETAILS}

\section{A. Model}

Liquid silica was modeled using a modified version of the popular van Beest-Kramer-van Santen (BKS) potential. ${ }^{65}$ The original BKS interatomic potential $U\left(r_{i j}\right)$ is of the form

$$
U_{\mathrm{BKS}}\left(r_{i j}\right)=\frac{q_{\mu} q_{\nu} e^{2}}{r_{i j}}+A_{\mu \nu} \mathrm{e}^{-b_{\mu \nu} r_{i j}}-\frac{C_{\mu \nu}}{r_{i j}^{6}},
$$

where $r_{i j}$ is the distance between an atom $i$ of type $\mu$ and an atom $j$ of type $v . e$ is the electronic charge and $q_{\mu}$ is the partial charge on atom $\mu$.

To avoid unphysical divergence of the $\mathrm{Si}-\mathrm{O}$ and $\mathrm{O}-\mathrm{O}$ pair potentials at small $r_{i j}$, we add a short-range repulsive pair potential ${ }^{59}$ of the form

$$
U_{\text {rep }}\left(r_{i j}\right)=4 \epsilon_{\mu \nu}\left[\left(\frac{\sigma_{\mu \nu}}{r_{i j}}\right)^{30}-\left(\frac{\sigma_{\mu \nu}}{r_{i j}}\right)^{6}\right] .
$$

$\epsilon_{\mu \nu}$ and $\sigma_{\mu \nu}$ are chosen such that the pair potential increases monotonically for distances $r_{i j}<r_{i j}^{*}$ (see Table I).

Accurate calculation of long-ranged interactions, such as electrostatic energies, under periodic boundary conditions remains a computationally demanding task. ${ }^{66}$ These energy terms are usually computed using Ewald summation. ${ }^{67}$ However, it has been argued that cancellation of electrostatic forces at long range means that in condensed systems the effective Coulomb interactions are actually rather short-ranged, ${ }^{68,69}$ allowing pairwise evaluation of the electrostatic energy with a spherical cutoff. We have employed a shifted truncation scheme similar to that of Wolf et al. ${ }^{70}$ to calculate these energies. Stable behaviour of geometry optimisation algorithms requires that the pair potential and associated force go
TABLE I. Parameters for the modified BKS pair potential used in this study. ${ }^{59,65} \mathrm{Si}-\mathrm{Si}$ interactions are electrostatic only (the parameters for the Buckingham, dispersive, and repulsive parts are all 0).

\begin{tabular}{lcc}
\hline \hline Parameter & Si-O & O-O \\
\hline$A_{\mu \nu}(\mathrm{eV})$ & 18003.7572 & 1388.7730 \\
$b_{\mu \nu}\left(\AA^{-1}\right)$ & 4.87318 & 2.76000 \\
$C_{\mu \nu}\left(\mathrm{eV} \AA^{6}\right)$ & 133.5381 & 175.0000 \\
$\epsilon_{\mu \nu}(\mathrm{eV})$ & $3.097948 \cdot 10^{-3}$ & $1.0510505 \cdot 10^{-3}$ \\
$\sigma_{\mu \nu}(\AA)$ & 1.313635 & 1.779239 \\
\hline$q_{\mu}$ & $\mathrm{Si}: 2.4$ & $\mathrm{O}: 1.2$ \\
\hline$r_{c} / \AA$ & 10.0 & \\
\hline \hline
\end{tabular}

smoothly to zero at the cutoff radius. ${ }^{19}$ We use an expression proposed by Fennell and Gezelter ${ }^{66}$ to calculate the Coulomb energy, replacing the first term in Eq. (1) with the following:

$$
U_{C}\left(r_{i j}\right)=q_{\mu} q_{\nu} \mathrm{e}^{2}\left(\frac{1}{r_{i j}}-\frac{1}{r_{c}}+\frac{1}{r_{c}^{2}}\left(r_{i j}-r_{c}\right)\right) .
$$

Here, $r_{c}$ is the cutoff radius.

Carre $e t a l{ }^{71}$ compared this truncation scheme with the Ewald summation for the BKS potential and found excellent agreement for both static and dynamic properties for cutoff radius $r_{c} \geq 10 \AA$. We have chosen $r_{c}=10 \AA$ to minimise the computational cost.

Similarly, a quadratic shift and cutoff at radius $r_{c}$ are introduced for the short-ranged Buckingham potential term in the BKS model. This scheme is analogous to that used by Stoddard and Ford for the Lennard-Jones potential. ${ }^{72}$ The second and third terms in Eq. (1) are replaced by

$$
\begin{aligned}
U_{\text {Buck }}= & A_{\mu \nu} \mathrm{e}^{-b_{\mu \nu} r_{i j}}-\frac{C_{\mu \nu}}{r_{i j}^{6}}+U_{\text {rep }}\left(r_{i j}\right)+\lambda_{1}+\lambda_{2} r_{i j}^{2}, \text { where } \\
\lambda_{1}= & -A_{\mu \nu} \mathrm{e}^{-b_{\mu \nu} r_{c}}\left(1+\frac{b_{\mu \nu} r_{c}}{2}\right)+\frac{4 C_{\mu \nu}}{r_{c}^{6}} \\
& +4 \epsilon_{\mu \nu}\left[-16\left(\frac{\sigma_{\mu \nu}}{r_{c}}\right)^{30}+4\left(\frac{\sigma_{\mu \nu}}{r_{c}}\right)^{6}\right] \\
\text { and } \lambda_{2}= & \frac{A_{\mu \nu} b_{\mu \nu}}{2 r_{c}} \mathrm{e}^{-b_{\mu \nu} r_{c}}-\frac{3 C_{\mu \nu}}{r_{c}^{8}}+4 \epsilon_{\mu \nu}\left[15 \frac{\sigma_{\mu \nu}^{30}}{r_{c}^{32}}-3 \frac{\sigma_{\mu \nu}^{6}}{r_{c}^{8}}\right] .
\end{aligned}
$$

The complete potential used in the present work is a combination of the shifted and truncated BKS potential [Eqs. (1) and (4)], the repulsive switching potential [Eq. (2)], and Coulombic interactions calculated by the Wolf method [Eq. (3)],

$$
U\left(r_{i j}\right)= \begin{cases}U_{\mathrm{Buck}}\left(r_{i j}\right)+U_{C}\left(r_{i j}\right)+U_{\mathrm{rep}}\left(r_{i j}\right), & \text { for } r_{i j}<r_{c}, \\ 0, & \text { otherwise }\end{cases}
$$

All parameters are given in Table I.

\section{B. Simulation details}

The dynamical data used in this study were obtained from microcanonical molecular dynamics (MD) simulations of bulk silica at a range of different energies. Periodic boundary conditions were employed, using a cubic simulation box containing 
TABLE II. Length of the MD trajectories at different temperatures. The equilibration time of $10^{6}$ MD steps ( $1 \mathrm{~ns}$ ) is excluded.

\begin{tabular}{lcc}
\hline \hline$T(\mathrm{~K})$ & MD steps & Length (ns) \\
\hline $2685,2902,3085,3207$ & $4 \times 10^{6}$ & 4 \\
3544,3854 & $2 \times 10^{6}$ & 2 \\
$4396,4821,5257,5752$ & $1 \times 10^{6}$ & 1 \\
\hline \hline
\end{tabular}

555 ions. The side length of the box was $20 \AA$, giving a fixed density of $2.3 \mathrm{~g} / \mathrm{cm}^{3}$. This density is close to the experimental value and has been studied in previous work. ${ }^{10,36}$

The qualitative behaviour of the diffusion constant, including the fragile-strong crossover, is the same in much larger systems, for example, in the study of Horbach and Kob using 8016 atoms. ${ }^{10}$ It has previously been shown $n^{53,73,74}$ that for BKS a system size of 100 particles is sufficient to avoid relevant finite-size effects for the configurational entropy and the relaxation dynamics.

Velocity-Verlet MD was performed with a time step of $1 \mathrm{fs}$. After an equilibration period of $1 \mathrm{~ns}$, trajectories were propagated until local ergodicity was obtained, as defined by the decay to zero of the Mountain-Thirumalai energy fluctuation metric. ${ }^{14,75}$ The length of time required for each trajectory is shown in Table II.

Because the BKS model is a simple empirical model, the characteristic temperatures may differ from their experimental values. In particular, the melting temperature is probably higher than the experimental value of $2000 \mathrm{~K} .{ }^{76}$ Nevertheless, many of the trajectories studied in this work are probably hotter than the melting temperature of BKS silica. Strong glass formers, which typically have very high melting points, exhibit many of the same dynamical anomalies as fragile glass formers even at temperatures well above the melting point. ${ }^{10}$ This behaviour is entirely consistent with dynamical theories of the glass transition that are independent of a thermodynamic transition. Following previous work, ${ }^{10,53,59,62,63}$ we consider the diffusive behaviour of silica in this temperature range in the same way that we have previously studied supercooled liquids.

\section{DIFFUSION ANALYSIS}

Figure 1 shows the temperature dependence of $\mathrm{Si}$ and O diffusion constants calculated using the Einstein formula $D=\lim _{t \rightarrow \infty} \frac{1}{6 t}\left\langle\mathbf{r}_{i}(t)^{2}\right\rangle$. At lower temperatures, $D(T)$ follows an Arrhenius relation $D(T)=D_{0} \exp \left(-E_{A} / k_{\mathrm{B}} T\right)$. We find $E_{A}=4.88 \mathrm{eV}$ for oxygen and $E_{A}=5.01 \mathrm{eV}$ for silicon (dashed lines). These activation energies are in good agreement with other simulations of BKS silica, e.g., Horbach et al. $(4.45 \mathrm{eV}$ for oxygen and $4.9 \mathrm{eV}$ for silicon $)^{60}$ and Saksaengwijit (4.84 eV for oxygen). ${ }^{53}$ Experimental measurements of diffusion coefficients in vitreous silica were reported by Mikkelsen $^{77}$ for oxygen atoms $\left(E_{A}=4.7 \mathrm{eV}\right)$ and Brebec et al. ${ }^{78}$ for silicon atoms $\left(E_{A}=6 \mathrm{eV}\right)$.

At higher temperatures, both silicon and oxygen diffusion show a clear non-Arrhenius curvature, beginning at around $3600 \mathrm{~K}$. This change corresponds to the "strong-fragile crossover" described in Sec. I C. The crossover occurs at

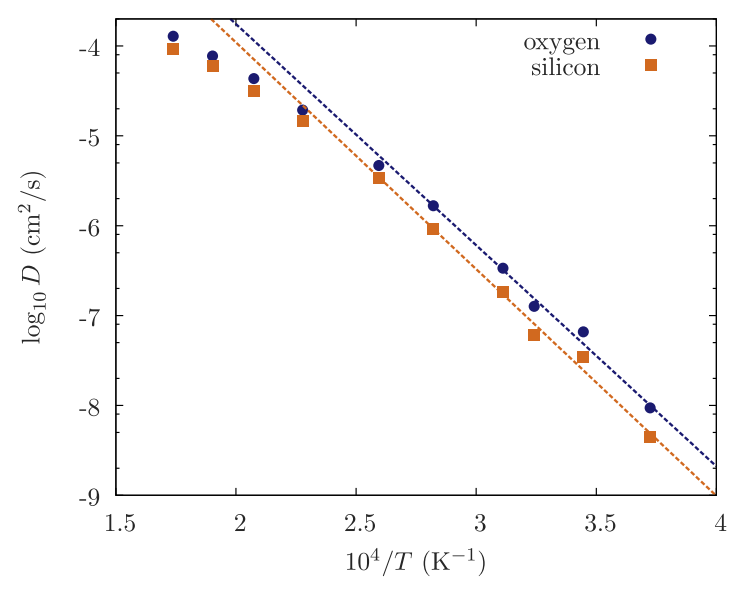

FIG. 1. Translational diffusion constants $D(T)$ for silicon and oxygen atoms plotted as a function of inverse temperature. The dashed lines represent Arrhenius fits to the low-temperature region of the data.

a slightly higher temperature in our model than in earlier work. $^{53,59,60}$

\section{A. Short-time diffusion constants}

In previous work, we have argued ${ }^{11-13}$ that the superArrhenius behaviour in fragile glass formers can be explained by increasingly negative correlation of particle displacements as the temperature decreases. The increased probability of particle velocity reversals leads to an increase in the effective free energy barrier to diffusion. The importance of negative correlation was demonstrated for the binary Lennard-Jones liquid ${ }^{11,12}$ and for ortho-terpheny ${ }^{13}$ by investigating the effect of the observation time scale on the apparent diffusion constants. In this contribution, we apply the same analysis to silica to identify whether significant correlation effects are also present for this strong glass former. The method is summarised here for convenience.

Locally ergodic MD trajectories for silica were divided into a series of short non-ergodic intervals of length $\tau$. The reduced diffusion constant $D(\tau)$ was calculated according to the Einstein relation, but replacing the average squared displacement of particle $i$ after time $t,\left\langle\mathbf{r}_{i}(t)^{2}\right\rangle$, with an effective mean squared displacement given by the following equation:

$$
\left\langle\mathbf{r}_{i}(t, \tau)^{2}\right\rangle=\left\langle\sum_{j=1}^{m} \Delta \mathbf{r}_{i}(j)^{2}\right\rangle,
$$

where $t=m \tau$ and $\Delta \mathbf{r}_{i}(j)=\mathbf{r}_{i}(j \tau)-\mathbf{r}_{i}((j-1) \tau)$. $j$ indexes the time intervals.

In Eq. (6), mean square displacements are evaluated directly over each short interval $j$ and added together to approximate the square displacement at longer times. This method neglects correlation between the displacement vectors of the particles in different time intervals.

Figure 2 shows the reduced-time diffusion constants for silica. In contrast to the equivalent graphs for BLJ and OTP, where small values of $\tau$ correspond to straight-line Arrhenius behaviour and larger values retrieve the super-Arrhenius curvature, for silica we observe positive curvature at small values of $\tau$. The straight-line behaviour expected for a strong glass former is recovered as $\tau$ increases. This result indicates that negative correlation of particle displacement is 


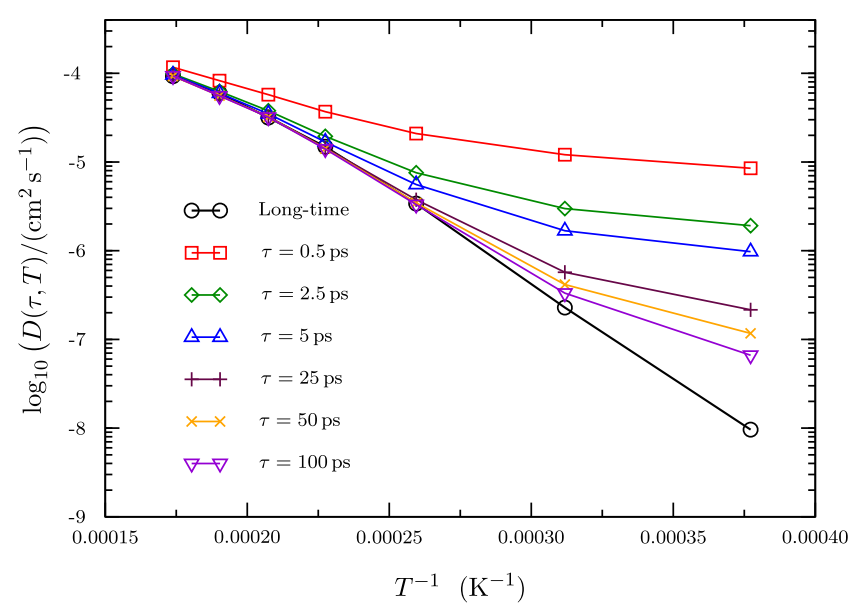

FIG. 2. Short-time diffusion constants $D(\tau, T)$ for silicon in liquid silica as a function of inverse temperature. Several values of the interval length $\tau$ are shown. The correct long-time diffusion constants calculated across the whole ergodic trajectory are shown for comparison. The equivalent plot for oxygen diffusion is qualitatively the same.

important for silica diffusion as well as for fragile glass formers, suggesting that their diffusive mechanisms are not qualitatively different.

The departure from Arrhenius behaviour for small $\tau$ occurs near the temperature for which $\tau \approx t_{p}$, where $t_{p}(T)$ is the upper limit of the plateau in the mean squared displacement that corresponds to particle caging (see Sec. I A). Therefore we believe that the curvature of the apparent diffusion constants is partly explained by the transition from caged motion to diffusive motion and indicates time heterogeneities in particle displacement vectors. On short time scales, the lowtemperature trajectories mostly sample small potential energy barriers within a particular set of nearest-neighbour cages, while the high-temperature trajectories are able to access a significant number of high energy barriers corresponding to cage-breaking motion. ${ }^{44,45}$ Because the average barrier to diffusion changes as a function of temperature, so does the gradient of the Arrhenius plot, leading to upwards curvature. If this interpretation of Fig. 2 is correct, then the same behaviour ought to be observed for any glass former (strong or fragile) at low enough temperatures and short enough time scales. Work is currently in progress to test this hypothesis.

It has previously been shown ${ }^{11-13}$ that using Eq. (6) to calculate diffusion constants neglects correlation between the different intervals over which the mean squared displacement is evaluated. The leading term excluded by this approximation depends only on the average angle $\theta_{j, j+1}$ between consecutive intervals of length $\tau$. As in previous work, we add this term back into correct the approximate mean-squared displacement used in Eq. (6). The correction factor is given by

$$
\begin{aligned}
\left\langle\mathbf{r}_{i}^{*}(t, \tau)^{2}\right\rangle & =\left\langle\sum_{j=1}^{m} \Delta \mathbf{r}_{i}(j)^{2}\right\rangle\left(1+2\left\langle\cos \theta_{j, j+1}\right\rangle\right), \\
D^{*}(T, \tau) & =D(T, \tau)\left(1+2\left\langle\cos \theta_{j, j+1}\right\rangle\right)
\end{aligned}
$$

Figure 3 shows both the corrected and uncorrected silicon diffusion constants $\left(D^{*}(T, \tau)\right.$ and $D(T, \tau)$, respectively) for selected values of $\tau$. $D^{*}(T, \tau)$ agrees much more closely

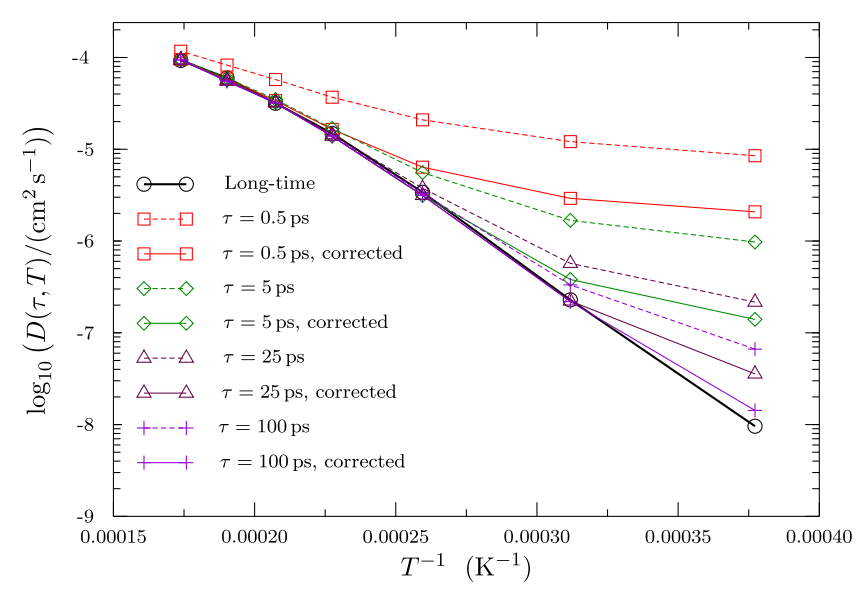

FIG. 3. Short-time Si diffusion constants for silica, corrected by re-addition of the leading neglected correlation term (see text). The long-time values and uncorrected short-time diffusion constants are shown for comparison.

than $D(T, \tau)$ with the correct long-time values, and the true Arrhenius temperature dependence is recovered at much smaller $\tau$.

For silica, as for BLJ ${ }^{11}$ and OTP, ${ }^{13}$ the correlation correction term becomes more negative as temperature decreases. In silica, this effect serves to remove the unphysical subArrhenius temperature dependence, whereas for the fragile systems, it was found to produce super-Arrhenius curvature.

These results suggest that diffusion in all glass formers is controlled by competition between the time scales of caging and negative correlations. When the latter time scale is very large compared to the former, negative correlations of particle displacements persist into the diffusive regime, increasing the effective energy barrier to diffusion and promoting negative curvature of the diffusion constants on an Arrhenius plot. Both time scales increase at low temperatures.

This interpretation argues that there is no fundamental difference between strong and fragile glass formers. Negative correlations are important for both, and in fragile liquids, they exist on very long time scales. For strong liquids, the correlation time scale is not much greater than the caging time.

In Secs. IV and V, we demonstrate a quantitative difference in time scales between the two types of glass formers to support this proposed description of supercooled dynamics.

\section{CAGE BREAKING}

Particles in supercooled liquids spend much of their time trapped within a cage of their nearest neighbours. Their motion may be separated into rapid rattling within a cage and slow diffusive transitions between cages. For BLJ ${ }^{30}$ and OTP, ${ }^{13}$ we have previously proposed microscopic definitions to classify rearrangements as "cage-breaking" or "non-cage-breaking." We have shown that cage breaks (CBs) reproduce the correct diffusion constants for fragile liquids in the moderately supercooled temperature regime. Cage-breaking rearrangements constitute a necessary and sufficient description of diffusive motion in these systems.

Here, we investigate whether the same definition of cage breaks is sufficient to capture the diffusive behaviour of our 
silica model, as a representative strong glass former. The definition is reproduced in outline in Secs. IV A-IV A 2, including details of the parameters used for silica. The silica trajectories are analysed in Sec. IV B.

\section{A. Defining a cage break in silica}

Cage-breaking events are associated with changes to the nearest-neighbour shell of a particle. Nearest neighbours of an atom are defined using a fixed cutoff distance, taken as the position of the first minimum in the $\mathrm{Si}-\mathrm{O}$ radial distribution function (RDF), $1.95 \AA$ (see Fig. 4). For an atom to leave the nearest neighbour shell during a rearrangement, it must move outside this radius and also move a distance greater than the "movement cutoff." This cutoff is set to $1.19 \AA$, the distance between the points where the first and second peaks in the $\mathrm{Si}-\mathrm{O} \mathrm{RDF}$ fall to $1 \%$ of their maximum height.

For BLJ systems, we required that an atom either lose or gain at least two of its neighbours to classify a transition as cage-breaking. However, particles in the BLJ system have 11 nearest neighbours on average, ${ }^{30}$ while in silica, a silicon atom has on average four oxygen nearest neighbours and an oxygen atom has two silicon nearest neighbours. Therefore the BLJ parameter values would be overly restrictive. Instead, we require that a particle changes at least half of its neighbours to be classified as cage-breaking (adding losses and gains together). Two neighbour changes are required for a silicon atom to be categorized as cage-breaking, and only one change is required for an oxygen atom.

\section{Reversed and productive cage breaks}

It is known from previous computational studies ${ }^{13,27,30}$ that intercage motion is negatively correlated on short time intervals and that most of this negative correlation arises from direct reversal of cage-breaking transitions. Following the method used for BLJ, ${ }^{30}$ we examine the total displacement of the cage-breaking atom over two consecutive cage breaks. If the net displacement is less than a threshold $d_{r e v}$, the second cage break is categorized as the return event of the first cage break. A series of cage breaks and their reversal events

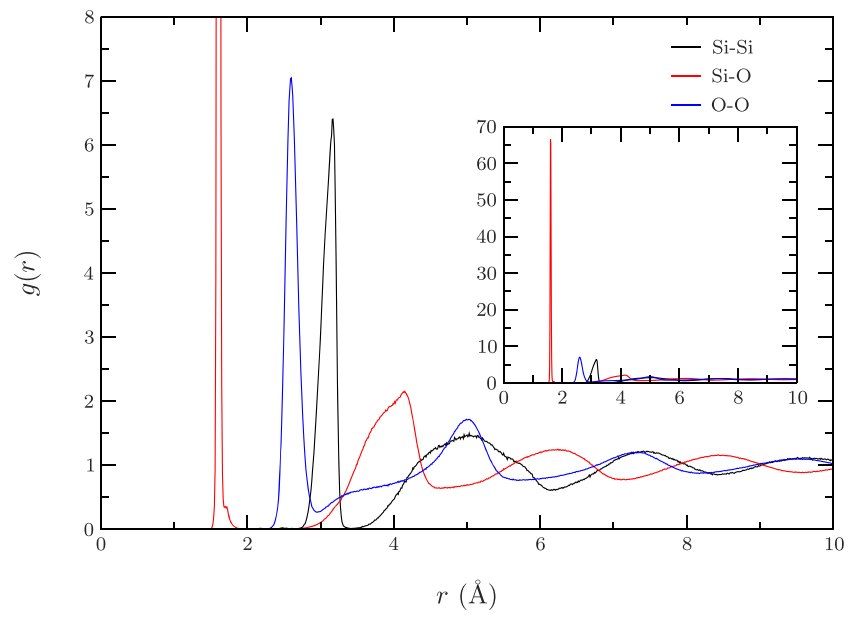

FIG. 4. Radial distribution functions for $\mathrm{Si}-\mathrm{Si}, \mathrm{Si}-\mathrm{O}$, and $\mathrm{O}-\mathrm{O}$ pairs, calculated from quenched configurations of a trajectory at $3207 \mathrm{~K}$. Consistency with higher temperature simulations has been verified. The inset represents the same plot with different axis scaling. is called a "reversal chain." During a simulation, these chains are recorded for every cage-breaking atom. Occasionally, the return event is not a cage break and instead takes place via several non-cage-breaking steps. These indirect reversals can also be identified by the occurrence of two consecutive identical cage breaks. ${ }^{30}$

For BLJ, the threshold displacement parameter is $d_{\text {rev }}=10^{-5} \sigma_{A A}^{2}$. However, the silica system we have studied is larger (555 atoms compared to 256) and has a lower number density $\left(0.069375 \AA^{-3}\right.$ compared to $\left.1.3 \sigma_{A A}^{-3}\right)$, so we expect that a larger $d_{r e v}$ will be required. Moreover, atoms in silica have far fewer nearest neighbours than in BLJ and so their "cages" are much more open, making it less likely that a reversed cage break will leave the atom within a small distance of its original position. After some experimentation, we chose $d_{\text {rev }}=10^{-2} \AA^{2}$. Qualitatively similar results are obtained using any $d_{\text {rev }}>10^{-4} \AA^{2}$, but $d_{\text {rev }}=10^{-2} \AA^{2}$ gives better quantitative agreement of the diffusion constants with the correct full-trajectory values.

\section{Calculating diffusion from cage breaks}

Identifying the nearest neighbours of an atom is complicated by vibration of atoms around locally favourable positions. This vibration can be removed by "quenching" structures to local minima of the potential energy landscape. Trajectories are analysed by extracting configurations of the system at regular intervals and performing local energy minimisation using the limited memory Broyden-Fletcher-GoldfarbShanno (LBFGS) algorithm. ${ }^{79,80}$ Quenching converts the trajectory into a sequence of jumps between local minima (often referred to as "inherent structures" in the glasses literature ${ }^{81}$ ). The frequency of minimum-to-minimum transitions decreases rapidly with temperature. The quenching interval was varied with the temperature of the trajectory to minimise the computational expense while still capturing the majority of minimum-to-minimum transitions.

The importance of cage-breaking motion for long-time diffusion in silica was investigated by computing effective diffusion constants using cage-breaking rearrangements only. The Einstein relation was used, but the square displacements obtained from real-time configurations during a MD trajectory were replaced by a sum of the square displacements made by cage-breaking atoms in the corresponding quenched trajectory,

$$
\mathbf{r}_{i}(t)^{2}=\sum_{i=1}^{N} \sum_{\mathrm{CBs}} \mathbf{r}_{\mathrm{CB}, i}^{2},
$$

where the sum over CBs includes all cage breaks for atom $i$ occurring before time $t$, and $\mathbf{r}_{\mathrm{CB}, i}$ refers to the displacement of the atom in a particular cage-breaking event.

Using Eq. (8) converts the continuous MD trajectory into a set of discrete cage-breaking jumps. All non-cage-breaking atomic motion is discarded. Initially, we assume that there is no correlation between the directions of particle jumps. We account for correlation by discounting all displacements from reversed cage breaks. All cage breaks in a reversal chain are discarded except the last one, and this one is only retained if the chain results in a net displacement. The remaining events are "productive cage breaks," and the sum in Eq. (8) is restricted 
to run over these events alone. We assume that, as we found for BLJ, the displacements of successive productive cage breaks of a particular atom are uncorrelated, and therefore removal of the direct reversal events is sufficient to account for correlation behaviour.

\section{B. Cage breaking results}

Figure 5 shows three sets of diffusion constants: those calculated from all cage-breaking rearrangements, those calculated from productive cage breaks only, and the correct long-time diffusion constants obtained from continuous MD trajectories.

The diffusion constants calculated from all CBs have broadly Arrhenius temperature dependence and overestimate the true diffusion constants across the entire temperature range.

Discounting reversed $\mathrm{CBs}$ reduces the calculated diffusion constants, leading to improved agreement with the fulltrajectory values in the strong regime below $3600 \mathrm{~K}$. However, using only productive cage breaks hardly affects the hightemperature diffusion constants because there are almost no reversed cage breaks detected at temperatures higher than $4000 \mathrm{~K}$.

Figure 5 demonstrates that productive cage breaks correctly reproduce translational motion in the strong temperature regime for liquid silica. This result is unsurprising since our definition of cage breaks for this system matches closely with intuitive definitions of bond breaking/forming and defect migration processes, which are known to dominate diffusive behaviour in silica. ${ }^{10,41,82,83}$ However, we have also shown that negatively correlated motion, here represented by reversed cage breaks, plays an important role in the strong regime but becomes negligible at temperatures above the fragile-tostrong crossover. At higher temperatures, the system has more energy and can access more rearrangement paths, so reversals are much less likely than at low temperatures. This analysis reinforces our observation in Sec. III A that negative correlations are important to produce Arrhenius behaviour of transport coefficients in supercooled liquids. Our findings also

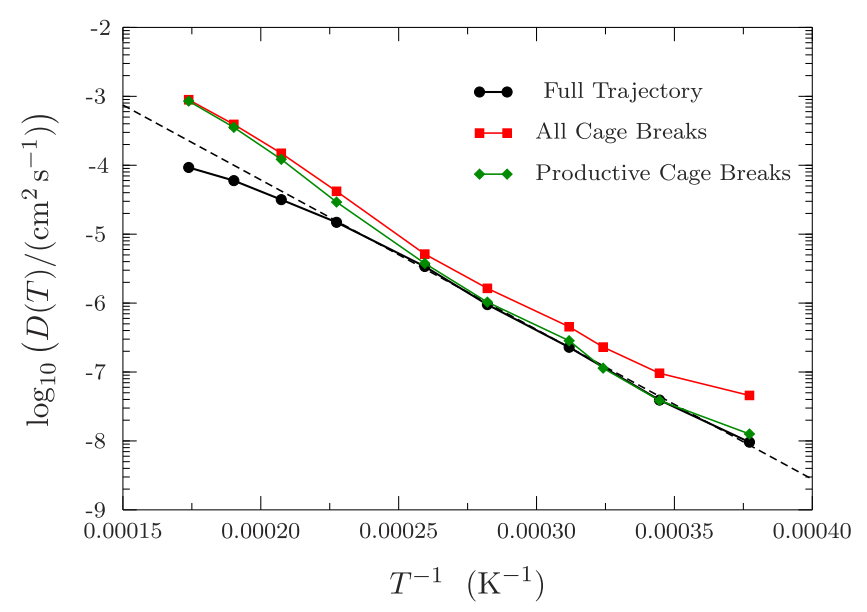

FIG. 5. Cage-breaking diffusion constants for silicon atoms in silica, calculated from the mean square displacements of productive cage breaks and of all cage breaks. Diffusion constants calculated from the full continuous MD trajectory are shown for comparison. The dashed line represents an Arrhenius fit to the full-trajectory values. The corresponding graph for $\mathrm{O}$ is qualitatively identical, and so is not shown. emphasise that the non-Arrhenius behaviour in the hightemperature regime for silica is qualitatively different from the super-Arrhenius curvature observed for fragile glass formers: in BLJ the fragile behaviour arises due to the presence of increasing negative correlations, ${ }^{11,12,30}$ but in the nonArrhenius regime for silica these correlations have little effect on the diffusion constants.

For silica, as for BLJ and OTP, ${ }^{13,30}$ diffusion constants calculated from cage breaks significantly overestimate the correct values at higher temperatures. This is because at high temperatures, the potential wells become increasingly anharmonic and atoms move further from their energy-minimum positions, ${ }^{43,84}$ and hence the description of the dynamics as a series of jumps between minima breaks down.

\section{Reversed cage breaks}

We have so far incorporated correlation effects into the cage-breaking model by restricting the calculation of diffusion constants to productive cage breaks alone. Alternatively, we may account for the correlations in an average fashion by taking the mean squared displacement from all cage breaks and applying a correction factor that depends upon the proportion of directly reversed cage breaks. This factor is analogous to the average correlation term in Eq. (7).

The "correction sum" is calculated using the reversal chains of a quenched trajectory, ${ }^{30}$

$$
c_{s}=\sum_{v} \sum_{z=1}^{l_{v}} \frac{(-1)^{z} \sum_{i} n_{i}^{v}(z)}{\sum_{i} M_{i}},
$$

where $v$ is an index running over all reversal chains in the trajectory and $M_{i}$ is the number of cage-breaking events for atom $i . l_{v}$ is the number of reversals in chain $v$ and $n_{i}^{v}(z)=l_{v}-z+1$ is the number of reversals after and including the $z$ th.

To use the correction sum, we make the approximation (justified below) that all cage breaks have squared displacement equal to the average value, $L$. The total squared displacement due to cage breaks is then given by ${ }^{30}$

$$
\left\langle\mathbf{r}(\text { total })^{2}\right\rangle=\sum_{i} M_{i} L\left(1+2 c_{s}\right) .
$$

We assume that $M_{i}=\left\langle M_{i}\right\rangle$ for all atoms.

Figure 6 shows that application of the correction term in Eq. (10) yields silicon diffusion constants that match those obtained from productive cage breaks very well. This result is expected when the assumptions of constant cage-breaking squared displacement $L$ and uniform $M_{i}=\left\langle M_{i}\right\rangle$ are valid. The results for oxygen are very similar and so they are omitted for brevity.

\section{Cage-breaking statistics}

Cage breaks have been shown to control the diffusive behaviour of both strong and fragile glass formers, so studying the properties of these events will provide new information on the dynamics of these systems. Cage breaks also provide us with characteristic time scales that we can compare between systems. 


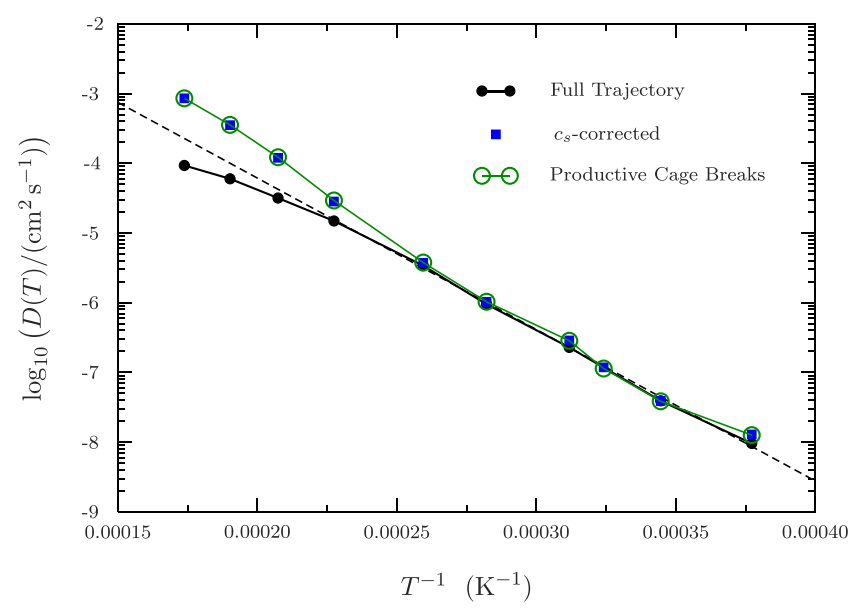

FIG. 6. Diffusion constants of silicon atoms in silica, calculated from the mean square displacements of all cage breaks corrected with the correlation sum $c_{s}$ and from productive cage breaks. The correct diffusion constants obtained from MD are shown for comparison. The dashed line represents an Arrhenius fit to the MD data.

Figure 7 shows that the number of cage breaks and reversals as a function of temperature exhibits approximate Arrhenius temperature dependence, but the absolute number of reversal events reaches a plateau at higher temperature. Above this temperature, the number of reversals becomes negligible compared to the number of cage breaks. The absolute number of reversal events decreases at lower temperatures, but the proportion of cage breaks that are reversed increases dramatically, showing again that it is important to consider correlation effects in displacements at low temperatures for strong glass formers as well as for fragile.

Figure 8 shows the average square displacement of a particle undergoing a cage break as a function of temperature. First, we note that the standard errors in this average are small relative to the size of the jump, which justifies the assumption made in Eq. (10) that all cage-breaking jump widths are equal at fixed temperature.

The temperature dependence of the jump widths is the first quantity where we observe a significant difference in

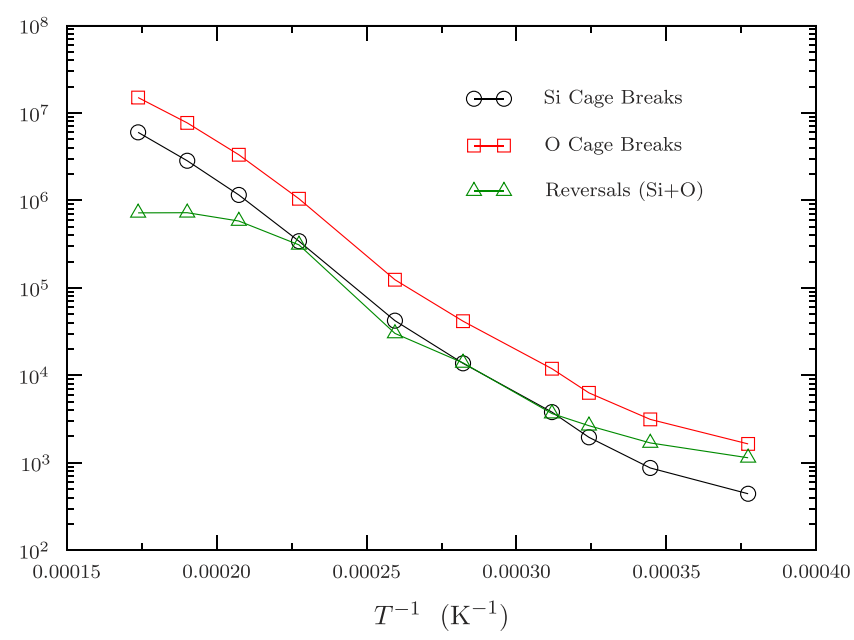

FIG. 7. Number of cage breaks and reversals as a function of temperature. These results have been normalised to a trajectory length of $1 \mathrm{~ns}$. The numbers of cage breaks are broken down into contributions from $\mathrm{Si}$ and $\mathrm{O}$ atoms, and the number of reversed cage breaks combines $\mathrm{Si}$ and $\mathrm{O}$.

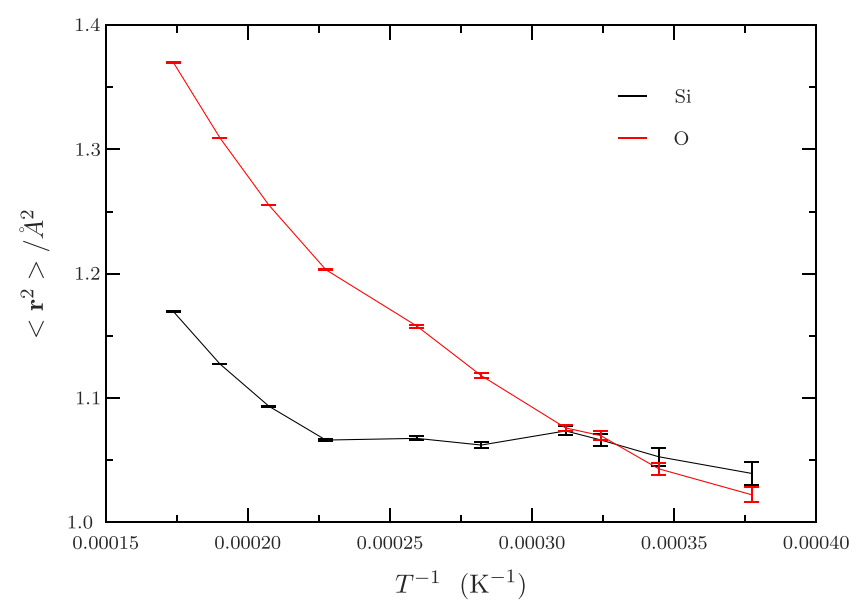

FIG. 8. Average square displacement in cage-breaking rearrangements for different atom types, as a function of inverse temperature. The error bars indicate the standard error in the mean square displacement. These errors are fairly small, particularly for high temperatures, so it is reasonable to approximate that all cage breaks at a particular temperature have equal squared displacement.

temperature dependence between the oxygen and silicon atoms. Horbach and $\mathrm{Kob}^{10}$ also found a difference between the oxygen and silicon atoms in their study of bond-breaking, when analysing the product of the bond lifetime and the diffusion constant.

Both atom types exhibit a significant increase in jump width on entering the high temperature non-Arrhenius regime. This increase probably arises from the breakdown of the assumption that the system is localised near potential energy minima, as discussed above. In the strong temperature regime, the silicon atoms show a fairly constant jump width, but the average squared displacement of oxygen cage breaks decreases with temperature. Horbach and $\mathrm{Kob}^{10}$ found that in this regime, the temperature dependence of oxygen diffusion followed the bond lifetime, but silicon diffusion slowed down more quickly. This may be evidence for the appearance of cooperative motion in the oxygen dynamics. ${ }^{63,85}$ Alternatively, there is evidence that rotational processes contribute to the long-range dynamics for oxygen but not for the silicon atoms and these processes become more relevant at lower temperatures. ${ }^{36}$ If an increasing fraction of oxygen cage breaks at lower temperatures are rotational processes, this could lead to a decreasing jump width but also retain a relationship between bond lifetime and diffusion.

\section{Comparing strong and fragile glass formers}

In Sec. III A we proposed that the difference between strong and fragile glass formers results from a difference in characteristic time scales. In this view, super-Arrhenius behaviour is caused by long-lasting negative correlations in particle motion, and pure Arrhenius behaviour corresponds to the limiting case where negative correlations occur only on short time scales. We expect that the correlation time in fragile liquids at low temperatures will greatly exceed the time scale on which particles are caged, but for strong liquids, this will not necessarily be the case.

The cage-breaking formalism allows us to probe these two time scales directly. The caging time scale may be estimated from the distribution of waiting times between consecutive cage breaks for an atom. This distribution shows 


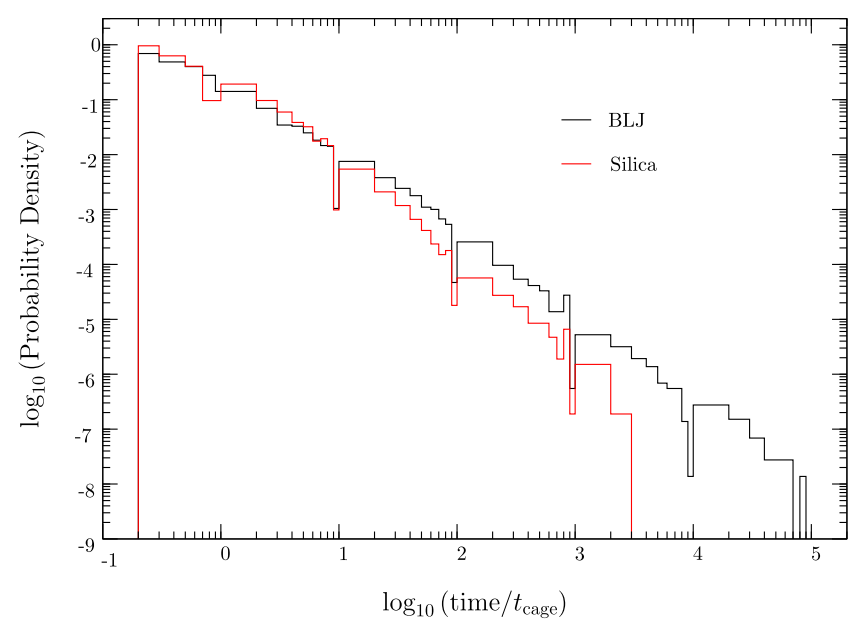

FIG. 9. Normalised histograms for the time spent in cage-breaking reversal chains for the silica trajectory at $3207 \mathrm{~K}$ and a 256-atom Kob-Andersen BLJ liquid with density $1.3 \sigma_{A A}^{-3}$ at temperature $0.65 \epsilon_{A A} / k_{\mathrm{B}}$. The data bins have unequal widths, for greater clarity on a log-log scale. Times are quoted in units of the characteristic caging time (see text) for each system.

approximately exponential decay with increased waiting time, so we have used the time constant of the distribution as the characteristic caging time.

To estimate the correlation time scale, we use the distribution of reversal chain times, defined as the length of simulation time elapsed between the start and end of a chain of reversed cage breaks (see Sec. IV A 1). Figure 9 shows histograms of this distribution for silica and for a comparable BLJ liquid, with time expressed in units of the characteristic caging time for each system.

Both probability distributions decay with increased chain time, with some chains persisting for hundreds or thousands of caging times. The BLJ histogram decays more slowly than the silica system and has more long chains. The longest chains for BLJ are at least an order of magnitude longer than the longest chains for silica.

These results show that the time scale of negative correlation in BLJ is appreciably longer than that for silica, which is consistent with our description of the differences between strong and fragile liquids. We note that the present measures of the two time scales suggest that even in silica, negative correlations persist to times significantly greater than the typical cage waiting time. This result may indicate that better characteristic times are called for, or that the exact ratio between these two time scales is less important than was suggested by the short-time diffusion analysis.

\section{POTENTIAL ENERGY LANDSCAPE ANALYSIS}

The concept of a potential energy landscape (PEL) was introduced in Sec. I B. To sample the landscape, we used an initial set of configurations taken from a quenched MD trajectory at $3207 \mathrm{~K}$ (see Sec. IV A). During the MD simulation, the coordinates of the system were saved at $100 \mathrm{fs}$ intervals and quenched. This procedure yields a sequence of local minima of the PEL in the order in which they were visited by the system. Since the initial MD trajectory is locally ergodic, the local minima obtained in this fashion should be representative of the region of configuration space available to the liquid. ${ }^{30,86,87}$
To calculate transition states on the PEL as well as minima, the OPTIM package ${ }^{88}$ was used. Using each pair of consecutive local minima as input, a discrete path was constructed, which is a sequence of transition states and the intermediate minima they connect.

OpтIM uses the doubly-nudged ${ }^{89,90}$ elastic band ${ }^{91,92}$ (DNEB) algorithm to construct an approximate minimumenergy pathway between a pair of known minima. The maxima on this pathway are candidate structures for transition states, which are accurately refined using the hybrid eigenvectorfollowing method. ${ }^{19,93}$ The minima connected by each transition state (TS) are found by stepping away from it parallel and antiparallel to the unique direction of negative curvature on the PEL and performing a local energy minimisation. These three steps (DNEB, TS refinement, and identification of local minima) make up one Oртім cycle. If gaps remain in the pathway between the two endpoint minima after the first cycle, a modified Dijkstra algorithm ${ }^{94}$ is employed to choose pairs of minima to connect in order to complete the discrete path in a small number of cycles. There are usually several physically relevant discrete paths between any given pair of minima. A single Oртім calculation attempts to identify one of these paths, but it is not guaranteed to find the lowest-energy or the fastest pathway.

Although the PEL for a system with constant volume is temperature independent, this sampling method is not. Depending on the mean temperature during the MD simulation, a different region of configuration space will be sampled. We used a trajectory at $3207 \mathrm{~K}$ because this temperature is well within the strong regime, but high enough that a moderate simulation length is sufficient to reach local ergodicity.

\section{A. Disconnectivity graph for liquid silica}

Disconnectivity graphs can be used to visualize a database of minima and transition states. ${ }^{19,95,96}$ The structure of a disconnectivity graph gives qualitative information about the topology of the landscape. Good structure-seeking systems, such as proteins that fold rapidly, have a single well-defined global minimum with other minima connected by low energy barriers. In contrast, glass formers have a "frustrated" landscape, meaning that there are many minima with similar energies separated by high barriers.

To construct a disconnectivity graph, minima are divided into mutually accessible sets, or "superbasins", at regularly spaced threshold energies. Mutual accessibility of two minima at an energy $E_{t}$ means that there exists at least one discrete path between them for which all transition states have $E<E_{t}$. The disconnectivity graph has energy as its vertical axis, and at each threshold $E_{t}$, every superbasin is represented by a single point, positioned on the horizontal axis for clarity. Each point is connected to a parent superbasin on the threshold energy level immediately above and to one or more daughter superbasins on the level below. Each branch terminates at the energy of a single minimum.

Figure 10 shows the disconnectivity graph for liquid silica. This is a highly frustrated landscape, with a wide range of minimum energies and barrier heights. The minima are grouped into local sets or "funnels," within which the barrier heights are relatively small $\left(\Delta E \leq k_{\mathrm{B}} T\right)$. Minima of different funnels 


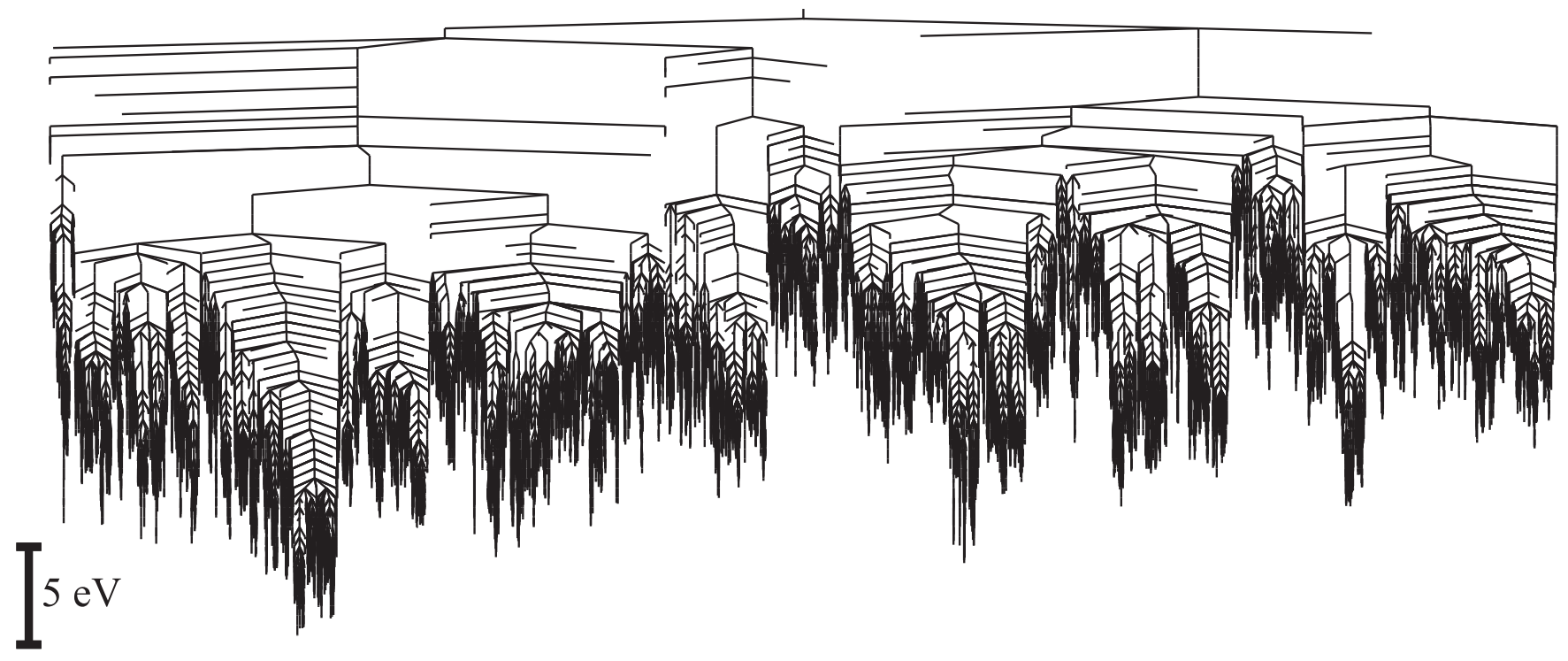

FIG. 10. Disconnectivity graph for liquid silica. The minima and transition states in this graph have been found by connecting each pair of adjacent minima in a quenched MD trajectory computed at $3207 \mathrm{~K}$.

are separated by significantly higher barriers with energies $\Delta E>>k_{\mathrm{B}} T$. As expected for a glass former, there is no unique lowest-energy region of the landscape ${ }^{44,45,97,98}$ As temperature decreases, the system becomes trapped in a particular local funnel on the experimental (and simulation) time scale and cannot explore other regions of the landscape.

In principle, the low-energy region corresponding to the crystal structure could be included in the disconnectivity graph, but it is very unlikely to be sampled on the simulation time scale.

Kushima et al. have previously reported a disconnectivity graph for silica, ${ }^{41}$ which appears qualitatively different to Fig. 10. The cause of this discrepancy is unclear, but it is probably due to differences in landscape exploration and optimisation methods.

\section{B. Cage-breaking analysis of the landscape}

Since it was shown in Sec. IV that cage-breaking rearrangements dominate the diffusive motion in silica, we can use the same definition of a cage break to determine which transition states in the energy landscape are significant for longtime diffusion. Each transition state in the landscape database may be classified as cage-breaking or otherwise, according to whether any atoms undergo a cage break between the two minima connected by this TS.

To visualise the difference between the two types of rearrangements, we take Fig. 10 and remove from it all transition states corresponding to cage-breaking TSs. This procedure causes the landscape to fragment into subgraphs, as connections between different nodes are removed. Figure 11 shows the fragments coloured according to the energy at which they become separated from the rest of the graph. Nearly every pair of minima in this graph appears as a separate fragment, meaning that most transitions between minima pass through a cage-breaking transition state.

The disconnectivity graph produced by excluding all noncage-breaking TSs in the same way is almost indistinguishable from the complete disconnectivity graph in Fig. 10, and hence is omitted. Removing non-cage-breaking transition states does not significantly affect the connectivity of the landscape, indicating that cage-breaking rearrangements are sufficient to access all regions of the PEL.

These results are qualitatively similar to those for fragile glass formers, but the degree to which the silica landscape becomes disconnected on removal of the cage-breaking transition states is greater than for BLJ or OTP. This observation suggests that cage-breaking motion is even more important for diffusion in silica than for diffusion in BLJ. This difference is not surprising because, as argued in Sec. IV B, our definition of cage breaks captures diffusive bond-breaking processes in silica very effectively.

\section{Geometric metabasin analysis}

In previous work, ${ }^{13,30}$ we have proposed a connection between cage breaks and the concept of metabasins (Sec. I B 1). Productive cage breaks, which are irreversible by construction and are essentially uncorrelated,$^{30}$ provide a geometric definition of metabasins on the PEL.

To identify metabasins, we use a "connected path" from the energy landscape database containing all the minima from the original quenched MD trajectory, together with all the intermediate minima on the discrete paths that connect them. This path is analogous to a quenched MD trajectory, except now all consecutive pairs of minima are linked by a single known transition state. Because the minima are time-ordered, reversals may be detected during the cage-breaking analysis, which allows productive cage breaks to be identified. All transition states are then classified as productive or otherwise according to whether they contain any productive cage breaks.

It is possible that if the same minimum is visited twice in the connected path, the coordinates may all be displaced slightly, complicating the identification of reversal events. To avoid this problem, we use the recently developed fast overlap method ${ }^{99}$ to align structures of the relevant 


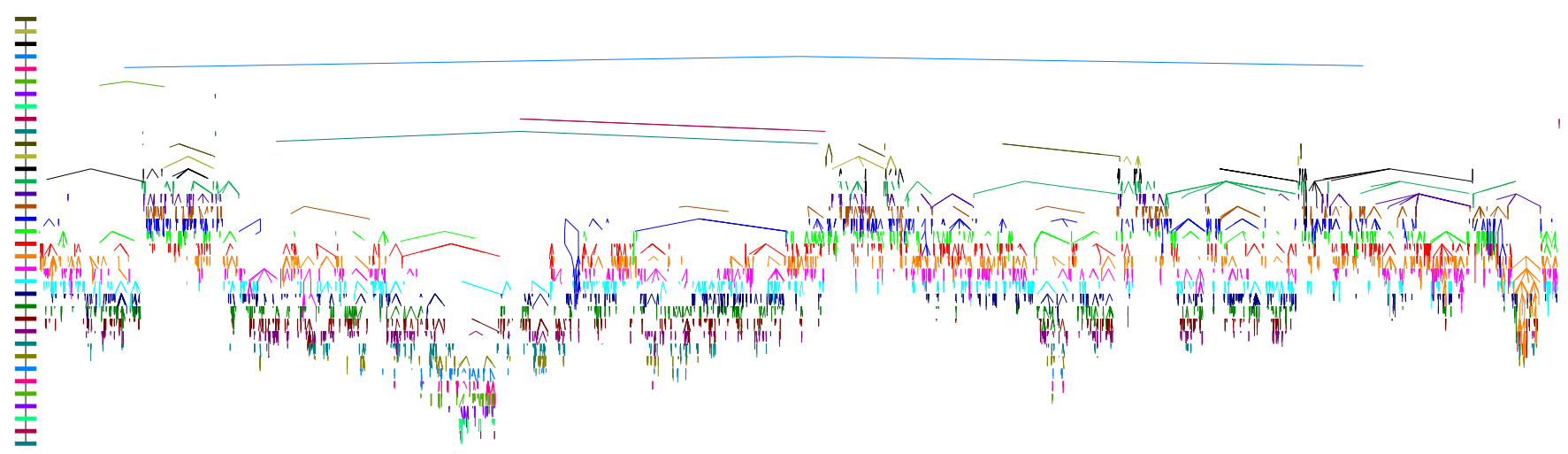

FIG. 11. Disconnectivity graph for liquid silica, similar to Fig. 10 but with only non-cage-breaking transition states included. The nodes are coloured according to the energy level at which they become disconnected from the rest of the graph. The colour associated with each energy level is indicated by the scale on the left. All fragments at a particular energy level are coloured the same, which is why bands of colour appear to run across the graph despite the presence of many fragments at each energy level. The separation of the energy levels is $1 \mathrm{eV}$.

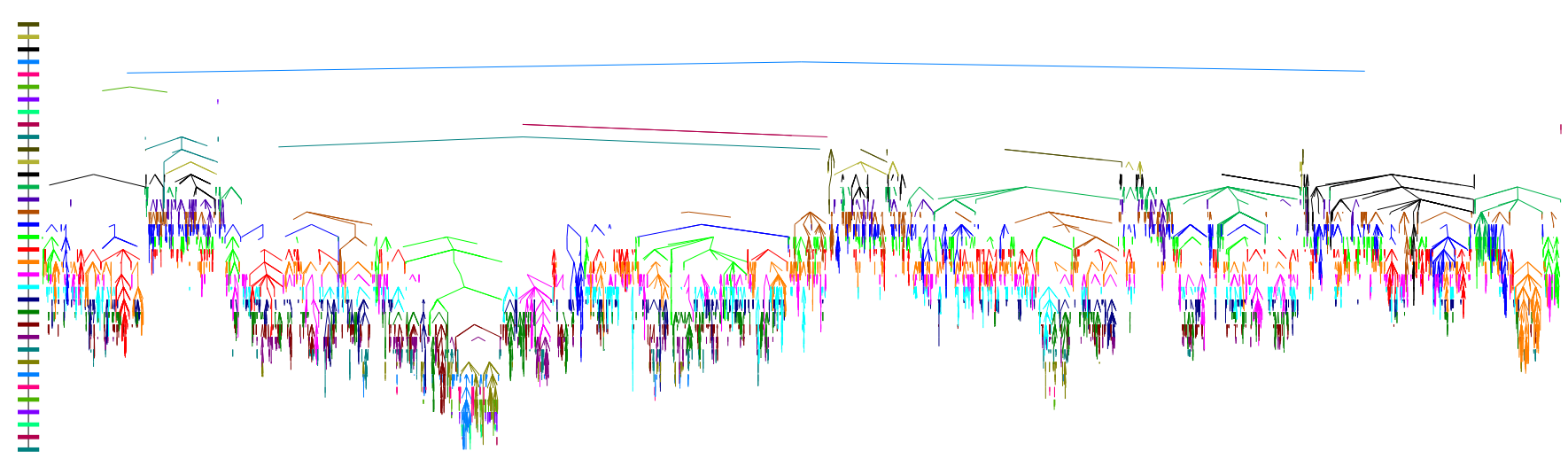

FIG. 12. Disconnectivity graph showing geometric metabasins for liquid silica. All transition states associated with a productive cage break have been excluded. The colour scheme is the same as in Fig. 11. The separation of the energy levels is $1 \mathrm{eV}$.

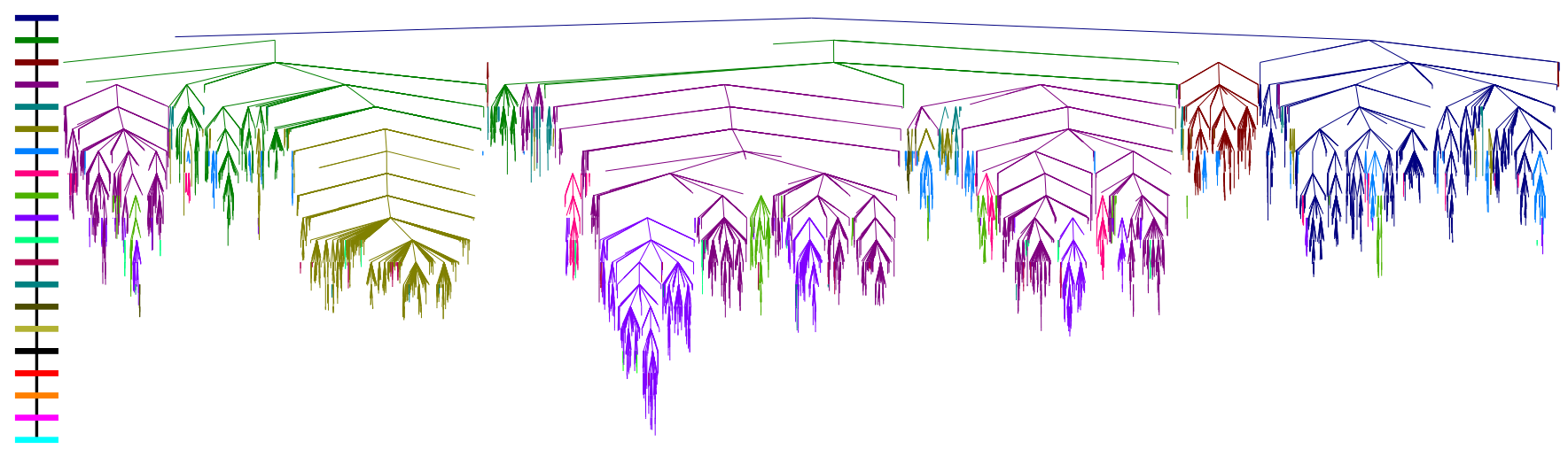

FIG. 13. Magnification from a larger disconnectivity graph showing geometric metabasins for the BLJ liquid. Productive cage-breaking transition states have been excluded. The colour scheme is the same as in Fig. 11. The separation of the energy levels is $2 \epsilon_{A A}$. Figure 12 contains 22995 minima, so this figure is restricted to showing $22995 \times 256 / 555=10606$ minima (rescaling according to the system size). The disconnectivity graph for the entire database is qualitatively identical.

minima before determining whether a reversal event has taken place.

Productive cage breaks are analogous to metabasin transition events, so a disconnectivity graph from which all productive transition states have been removed should fragment into regions that correspond to geometric metabasins. Figure 12 shows these metabasins for silica. Figure 13 shows a disconnectivity graph for the BLJ fluid produced in exactly the same way, for comparison. See Sec. VI for details of the BLJ system.

The metabasin disconnectivity graph is less fragmented than the non-cage-breaking graph, Fig. 11, because transition states within a metabasin comprise both non-cage-breaking and reversed cage-breaking transition states. However, Fig. 12 
is still highly fragmented and contains few clearly defined metabasins. The metabasins for silica are small compared to BLJ and contain fewer high-energy transition states. It has been suggested ${ }^{46,100}$ that the difference between strong and fragile liquids is related to the size of the metabasins: fragile liquids have large metabasins and significant hierarchical ordering of the landscape, whereas the landscapes of strong liquids exhibit structure on a single length scale only. Our analysis, like that of Heuer et al., suggests that metabasins are present in strong glass formers. However, when we compare to fragile liquids, the metabasins are significantly smaller and may be less important for the overall dynamics.

\section{ENERGY LANDSCAPES FOR DIFFERENT CLASSES OF GLASS FORMERS}

In this section, we compare some simple global properties of the landscapes of strong (silica) and fragile $\left(\mathrm{BLJ}^{30}\right)$ liquids. Full details of the simulation methods used to sample the PEL for BLJ may be found in previous work. ${ }^{30}$ The parameter set corresponds to the popular Kob-Andersen model, ${ }^{9}$ and the system was studied at a density of $1.3 \sigma_{A A}^{-3}$, with a simulation box containing 256 atoms (204 of type A and 52 of type B).

The silica database in Sec. V was obtained from a trajectory at $3207 \mathrm{~K}$, near the mode coupling critical temperature for this $\operatorname{system}\left(T_{c} \approx 3330 \mathrm{~K}^{10}\right)$. To give the most accurate comparison possible, we sampled the BLJ landscape using a trajectory at $T=0.65 \epsilon_{A A} / k_{\mathrm{B}}$, close to the fitted value of the modecoupling temperature for this density, ${ }^{101} T_{c}=0.66 \epsilon_{A A} / k_{\mathrm{B}}$. The MD time step was $0.005\left(m \sigma_{A A}^{2} / \epsilon_{A A}\right)^{1 / 2}$ and the simulation was run for $5 \times 10^{5}\left(m \sigma_{A A}^{2} / \epsilon_{A A}\right)^{1 / 2}$ to reach local ergodicity.

Different trajectory lengths were required to reach local ergodicity for the two systems, so the two databases contain very different numbers of stationary points. The silica database contains 22995 minima and 24237 transition states, while the BLJ database contains 152913 minima and 184648 transition states. However, since both databases were constructed from a locally ergodic MD trajectory, they should each provide a faithful representation of the region of configuration space explored by the liquid.

\section{A. Simple landscape metrics}

Figure 14 shows normalised distributions of the energies of minima for silica and BLJ. For each database, the energies are expressed relative to the lowest-energy minimum and given in units of $k_{\mathrm{B}} T$. Both distributions are approximately Gaussian, as expected. ${ }^{42}$

The BLJ database has a larger mean and standard deviation of minimum energies than the silica database, indicating that this landscape is rougher, with deeper metabasins and hence a wider range of energies to explore. Moreover, the BLJ distribution deviates more from Gaussian behaviour, exhibiting slight positive skew and a non-Gaussian tail at the low-energy end.

Saksaengwijit et al. have argued for the existence of a cutoff in the distribution of minimum energies for strong liquids,

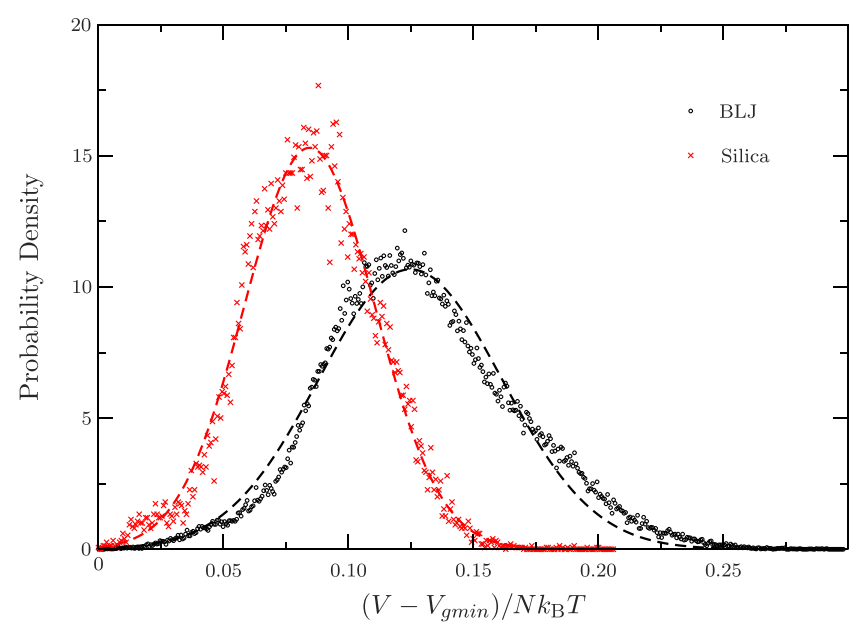

FIG. 14. Normalised distributions of the energies of minima in the landscape databases for BLJ and silica. Energies are expressed relative to the global minimum energy in each case and are given in units of $k_{\mathrm{B}} T$ per particle to allow comparison between the different systems. Dashed lines show Gaussian fits to the distributions of the corresponding colour.

below which there exist many fewer states than the Gaussian distribution would predict. ${ }^{42}$ We do not see such a cutoff in Fig. 14 because our system is too large and probably contains multiple weakly interacting subsystems: the effect of the cutoff in each subsystem is smeared out when they are combined. ${ }^{42}$ However, the silica distribution has a smaller low-energy tail than the BLJ distribution, which may be a signature of the low-energy cutoff in these larger systems.

Figure 15 shows normalised histograms of the barrier heights in the two databases, with the same energy scaling as before. Barrier heights are defined as the energy difference between a minimum and an adjacent transition state. Both histograms show an exponential decrease in probability density with increasing barrier height, with a super-exponential excess of small energy barriers. Although the two systems have comparable temperatures, the distribution of energy barriers sampled by the BLJ fluid is slightly wider than that encountered by silica. This difference again indicates a rougher landscape for BLJ, corresponding to larger metabasins.

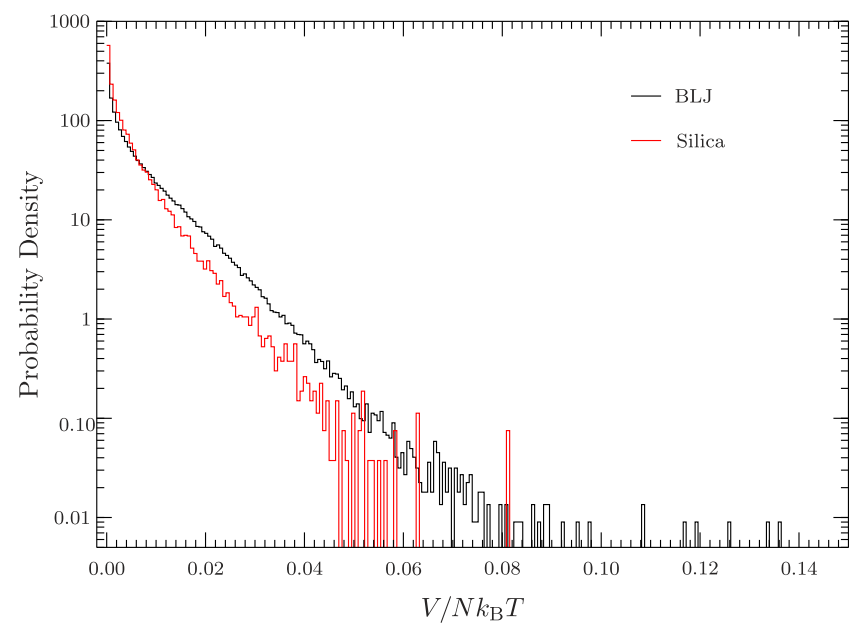

FIG. 15. Normalised histograms of the elementary barrier heights in the landscape databases for BLJ and silica. Barriers are expressed in units of $k_{\mathrm{B}} T$ per particle to allow comparison between the different systems. 


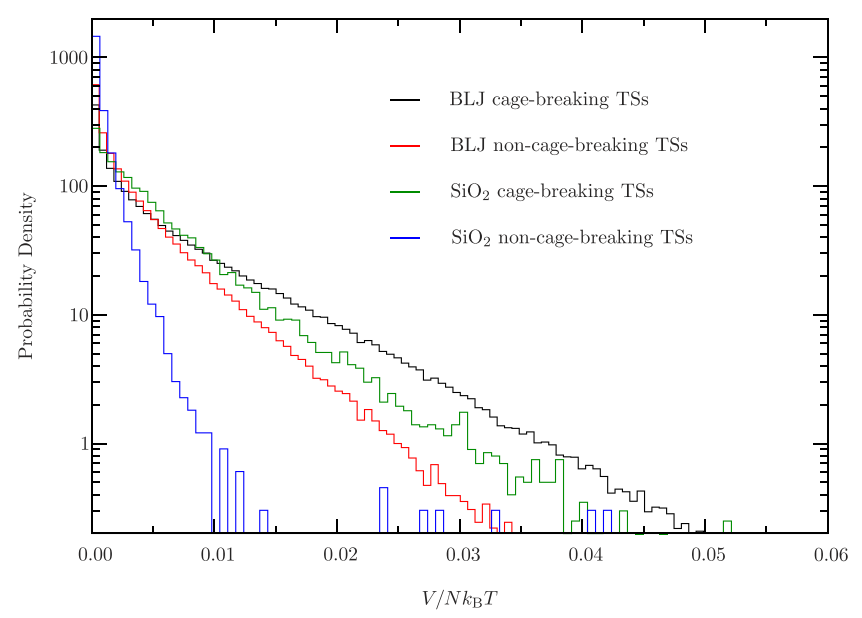

FIG. 16. Normalised histograms of the elementary energy barrier heights corresponding to cage-breaking and non-cage-breaking transitions in BLJ and silica. Barriers are expressed in units of $k_{\mathrm{B}} T$ per particle to allow comparison between the different systems. The histogram is truncated at low probabilities to mitigate the difference in resolution of the BLJ and silica histograms arising from the different database sizes.

In Fig. 16, we decompose the barrier height distributions into separate histograms for cage-breaking and non-cagebreaking transition states using the definition described in Sec. IV A. As expected, cage-breaking barriers are generally higher than non-cage-breaking. However, we also see a much greater difference between characteristic cage-breaking and non-cage-breaking barrier heights for silica than we do for BLJ. This result quantifies our earlier statement that cage-breaking rearrangements dominate the high-barrier processes more in silica than in BLJ. It is plausible that this represents a general difference between strong and fragile glass formers: fragile liquids exhibit a significant amount of intracage motion alongside cage-breaks, whereas for strong liquids, cage breaks account for nearly all of the particle motion.

\section{B. Frustration metric}

We use a recently proposed metric to quantify the degree of frustration in the potential energy landscapes of the two glass formers. ${ }^{102}$ Here, "frustration" describes the existence of competing low-lying potential energy minima separated by high barriers. Highly frustrated landscapes have many such minima, which makes relaxation to the equilibrium zero-temperature structure relatively slow. Good structure-seekers have low frustration, and glass formers have high frustration. ${ }^{102}$ The frustration metric facilitates comparison of PELs for very different systems.

The true global minimum of a glass former is the crystal structure, which is intentionally excluded from our databases. Hence the frustration index expresses the ability of the system to locate the lowest-energy amorphous minimum in our database.

Figure 17 presents the frustration index ${ }^{102} f$ for the two systems as a function of temperature. As temperature decreases, the ability to cross high barriers decreases and frustration increases. At very low temperatures, the equilibrium occupation probability of the global minimum dominates and so frustration decreases. However, since glass-formers are

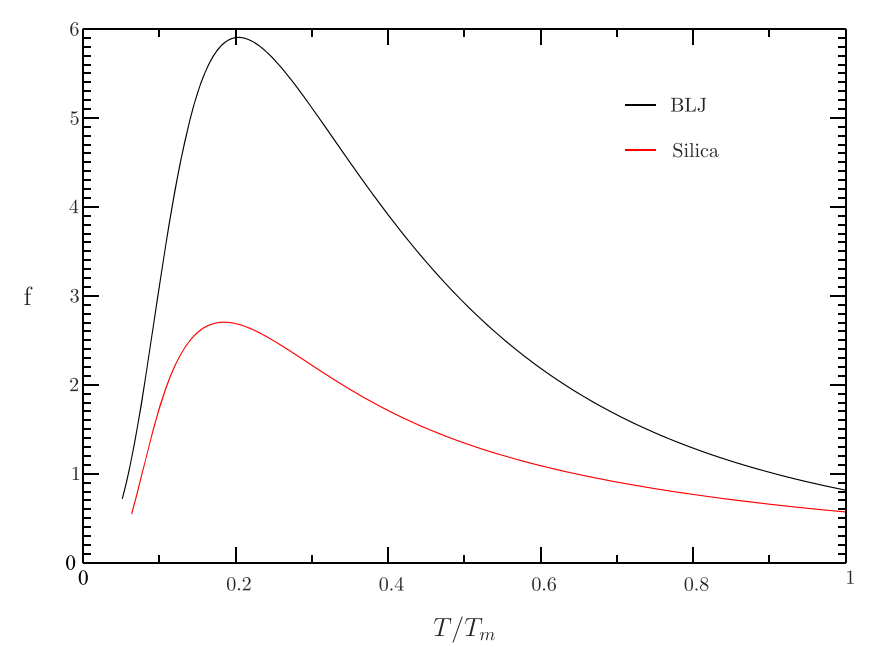

FIG. 17. Frustration index ${ }^{102}$ for BLJ and silica as a function of $T / T_{m} . T_{m}$ is the melting temperature determined from a peak in the constant volume heat capacity calculated using the harmonic superposition approximation. ${ }^{19}$

inevitably out of equilibrium at these low temperatures, this region of the figure is not relevant to supercooled liquids.

Both liquids have values of $f$ in the characteristic range for multi-funnel energy landscapes, ${ }^{102}$ as expected for glass formers. The silica database falls near the bottom of this range and is less frustrated than the BLJ database at all temperatures. This difference is consistent with the notion that the landscapes of fragile glass formers are dominated by large metabasins while strong glass formers have a more uniform organisation with small metabasins and less frustration. ${ }^{46}$

The disconnectivity graphs of Sec. V and all the landscape metrics studied in this section show that there is no major qualitative difference between the landscapes of silica and BLJ, but that the latter system has a slightly rougher PEL with larger metabasins. The metabasin disconnectivity graph and the frustration index provide clearer evidence for a difference between the PEL of strong and fragile glass formers than the other simple metrics we have studied, probably because these are the only two measures we have considered that account for the topology and the connectivity of the landscape. Changes in connectivity of minima as a function of trajectory temperature were previously found to be an important factor in the superArrhenius behaviour of BLJ. ${ }^{33}$ The role of landscape topology in producing negative correlation behaviour and controlling diffusion may be amenable to further analysis by describing the landscape as a network.

\section{CONCLUSIONS}

We have studied the diffusive behaviour of the BKS model for viscous silica, using a number of analytical techniques developed previously for fragile glass formers.

We have shown that the bond-breaking and forming processes that dominate long-time diffusion in low-temperature silica may be accurately described by the same definition of cage-breaking rearrangements that describes diffusion in the BLJ liquid, even though cages in silica are much less compact than BLJ and atomic coordination numbers are much smaller.

The cage-breaking model fails to reproduce the correct diffusion constants at higher temperatures, as also seen for BLJ 
and OTP. At high temperatures, the system wanders further from the bottom of the potential energy wells, ${ }^{43}$ complicating the identification of cage breaks. This failure occurs close to the temperature at which non-Arrhenius behaviour emerges in the diffusion constants. The relationship between these two phenomena will be explored in future work.

The cage-breaking results and the short-time effective diffusion constants calculated in Sec. III A both indicate that the presence of negative correlations is fundamentally important to transport processes in silica. We have shown that negative correlations in the particle displacement vector over time scales comparable with the caging time are associated with strong behaviour. This result is striking because the same correlation effect causes super-Arrhenius behaviour in fragile liquids.

We hypothesise that the difference between the two types of glass formers is that negative correlations in fragile systems persist over much longer time scales than the caging time scale and hence influence the diffusion constants more than for silica. The longer correlation time of fragile liquids may be caused by cooperative motion, ${ }^{85,87,103}$ which is much less prevalent in silica than in fragile glass formers. ${ }^{62,63}$ This interpretation would suggest that a continuous spectrum of fragility is controlled by the competition between the caging and correlation time scales. We have attempted to quantify these time scales by investigating chains of correlated cagebreaking rearrangements. We find that both strong and fragile liquids exhibit many chains with lengths greater than the characteristic cage waiting time, but that chains in the BLJ fluid are significantly longer on average than those in silica.

To investigate the origin of the correlation effects, we studied the potential energy landscape of silica, which was found to be similar in many respects to that of BLJ. Both systems have a complex multi-funneled landscape with large energy barriers separating low-lying amorphous energy minima. However, several key differences between the two models were identified. The separation of energy scales between cagebreaking and non-cage-breaking energy barriers is greater for silica than for BLJ, emphasising the greater importance of cage breaks for the strong liquid. The connectivity of the landscape is almost completely destroyed when cage-breaking transition states are removed, but is unaffected when non-cage breaks are removed. Cage breaks are both necessary and sufficient to traverse the energy landscape and hence are required for dynamical processes.

Geometrical metabasins for silica are smaller than for BLJ. This observation is in line with previous predictions regarding the difference between the energy landscapes of strong and fragile liquids. ${ }^{46,100}$ By definition, transitions between metabasins correspond to an uncorrelated randomwalk process, so correlation only exists within a metabasin and hence smaller metabasins mean less correlation in minimumto-minimum transitions. This conclusion is consistent with our argument that correlation times in fragile liquids are longer relative to the caging time scale than in strong liquids.

Finally, we found that the potential energy landscape of silica is less frustrated than for BLJ. This difference was shown by several landscape properties, notably by a frustration metric which measures the relative height of energy barriers between minima of similar energies. It is interesting that the two measures distinguishing most clearly between strong and fragile landscapes (i.e., metabasins and the frustration metric) are those that take greatest account of the connectivity and topology. Connectivity is likely to be crucial in understanding the origins of negative correlation behaviour in glass formers. ${ }^{33}$

Our results indicate that there is no sharp distinction between some liquids that are strong and others that are fragile. The negative correlation behaviour that gives rise to superArrhenius diffusion in fragile liquids is still present in silica, but to a lesser extent. We have shown that there is no qualitative difference in the underlying potential energy landscapes of strong and fragile glass formers, but we diagnose quantitative variation in metrics related to landscape topology and connectivity. These findings add to the growing body of evidence that strong glass formers represent one extreme of a continuous spectrum of fragilities. ${ }^{61-64}$

\section{ACKNOWLEDGMENTS}

This work was supported by the University of Cambridge through a CHSS studentship to S.P.N., by EPSRC grant EP/N035003/1, and by the ERC. M.B. wishes to acknowledge an Erasmus+ fellowship. Please see https:// doi.org/10.17863/CAM.13646 for the dataset containing data, source code, and documentation required to reproduce the results contained within this article.

${ }^{1}$ H. Vogel, Z. Phys. 22, 645 (1921).

${ }^{2}$ G. S. Fulcher, J. Am. Ceram. Soc. 8, 339 (1925).

${ }^{3}$ G. Tammann and W. Hesse, Z. Anorg. Allg. Chem. 156, 245 (1926).

${ }^{4}$ C. A. Angell, J. Non-Cryst. Solids 131-133, 13 (1991).

${ }^{5}$ C. A. Angell, Science 267, 1924 (1995).

${ }^{6}$ C. A. Angell, K. L. Ngai, G. B. McKenna, P. F. McMillan, and S. W. Martin, J. Appl. Phys. 88, 3113 (2000).

${ }^{7}$ T. A. Vilgis, Phys. Rev. B 47, 2882 (1993).

${ }^{8}$ N. Mauro, M. Blodgett, M. Johnson, A. Vogt, and K. Kelton, Nat. Commun. 5, 4616 (2014)

${ }^{9}$ W. Kob and H. Andersen, Phys. Rev. E 51, 4626 (1995).

${ }^{10}$ J. Horbach and W. Kob, Phys. Rev. B 60, 3169 (1999).

${ }^{11}$ V. K. de Souza and D. J. Wales, Phys. Rev. B 74, 134202 (2006).

${ }^{12}$ V. K. de Souza and D. J. Wales, Phys. Rev. Lett. 96, 057802 (2006).

${ }^{13}$ S. Niblett, V. de Souza, J. Stevenson, and D. Wales, J. Chem. Phys. 145, 024505 (2016).

${ }^{14}$ V. K. de Souza and D. J. Wales, J. Chem. Phys. 123, 134504 (2005).

${ }^{15}$ B. Doliwa and A. Heuer, Phys. Rev. Lett. 80, 4915 (1998).

${ }^{16}$ B. Doliwa and A. Heuer, J. Phys.: Condens. Matter 11, A277 (1999).

${ }^{17}$ E. R. Weeks and D. A. Weitz, Phys. Rev. Lett. 89, 095704 (2002).

${ }^{18}$ E. R. Weeks and D. Weitz, Chem. Phys. 284, 361 (2002).

${ }^{19}$ D. J. Wales, Energy Landscapes (Cambridge University Press, Cambridge, 2003).

${ }^{20}$ W. Götze and L. Sjögren, J. Phys. C 21, 3407 (1988).

${ }^{21}$ P. Chaudhuri, L. Berthier, and W. Kob, Phys. Rev. Lett. 99, 060604 (2007).

${ }^{22}$ R. Pastore, A. Coniglio, and M. Pica Ciamarra, Soft Matter 10, 5724 (2014).

${ }^{23}$ E. Rabani, J. D. Gezelter, and B. J. Berne, J. Chem. Phys. 107, 6867 (1997).

${ }^{24}$ E. Rabani, J. D. Gezelter, and B. J. Berne, Phys. Rev. Lett. 82, 3649 (1999).

${ }^{25}$ E. Rabani, J. D. Gezelter, and B. J. Berne, J. Chem. Phys. 110, 3444 (1999).

${ }^{26}$ E. Rabani, J. D. Gezelter, and B. J. Berne, Phys. Rev. Lett. 85, 467 (2000).

${ }^{27}$ K. Vollmayr-Lee, J. Chem. Phys. 121, 4781 (2004).

${ }^{28}$ M. Kluge and H. R. Schober, Phys. Rev. B 70, 224209 (2004).

${ }^{29}$ C. Gaukel and H. Schober, Solid State Commun. 107, 1 (1998). 
${ }^{30}$ V. K. de Souza and D. J. Wales, J. Chem. Phys. 129, 164507 (2008).

${ }^{31}$ R. Candelier, A. Widmer-Cooper, J. K. Kummerfeld, O. Dauchot, G. Biroli, P. Harrowell, and D. R. Reichman, Phys. Rev. Lett. 105, 135702 (2010).

${ }^{32}$ J. Helfferich, F. Ziebert, S. Frey, H. Meyer, J. Farago, A. Blumen, and J. Baschnagel, Phys. Rev. E 89, 042603 (2014).

${ }^{33}$ V. K. de Souza and D. J. Wales, J. Chem. Phys. 130, 194508 (2009).

${ }^{34}$ A. Heuer, J. Phys.: Condens. Matter 20, 373101 (2008).

${ }^{35} \mathrm{~J}$. Helfferich, K. Vollmayr-Lee, F. Ziebert, H. Meyer, and J. Baschnagel, Europhys. Lett. 109, 36004 (2015).

${ }^{36}$ A. Saksaengwijit and A. Heuer, Phys. Rev. E 74, 051502 (2006).

${ }^{37}$ M. Goldstein, J. Chem. Phys. 51, 3728 (1969).

${ }^{38}$ S. Sastry, P. G. Debenedetti, and F. H. Stillinger, Nature 393, 554 (1998).

${ }^{39}$ W. Kob and H. C. Andersen, Phys. Rev. Lett. 73, 1376 (1994).

${ }^{40}$ H. Jónsson and H. C. Andersen, Phys. Rev. Lett. 60, 2295 (1988).

${ }^{41}$ A. Kushima, X. Lin, J. Li, X. Qian, J. Eapen, J. C. Mauro, P. Diep, and S. Yip, J. Chem. Phys. 131, 164505 (2009).

${ }^{42}$ A. Saksaengwijit, J. Reinisch, and A. Heuer, Phys. Rev. Lett. 93, 235701 (2004).

${ }^{43}$ P. Jund and R. Jullien, Phys. Rev. Lett. 83, 2210 (1999).

${ }^{44}$ T. F. Middleton and D. J. Wales, J. Chem. Phys. 118, 4583 (2003).

${ }^{45}$ T. F. Middleton and D. J. Wales, Phys. Rev. B 64, 024205 (2001).

${ }^{46}$ F. H. Stillinger, Science 267, 1935 (1995).

${ }^{47}$ B. Doliwa and A. Heuer, Phys. Rev. E 67, 030501(R) (2003).

${ }^{48}$ T. Keyes and J. Chowdhary, Phys. Rev. E 64, 032201 (2001).

${ }^{49}$ T. F. Middleton and D. J. Wales, J. Chem. Phys. 120, 8134 (2004).

${ }^{50}$ B. Doliwa and A. Heuer, Phys. Rev. E 67, 031506 (2003).

${ }^{51}$ B. Doliwa and A. Heuer, J. Phys.: Condens. Matter 15, S849 (2003).

${ }^{52}$ S. Büchner and A. Heuer, Phys. Rev. Lett. 84, 2168 (2000).

${ }^{53}$ A. Saksaengwijit and A. Heuer, Phys. Rev. E 73, 061503 (2006).

${ }^{54}$ S. Sastry, Nature 409, 164 (2001).

${ }^{55}$ L. V. Woodcock, C. A. Angell, and P. A. Cheeseman, J. Chem. Phys. 65, 1565 (1976).

${ }^{56}$ K. U. Schug, H. E. King, Jr., and R. Böhmer, J. Chem. Phys. 109, 1472 (1998).

${ }^{57}$ K.-U. Hess, D. Dingwell, and E. Rössler, Chem. Geol. 128, 155 (1996).

${ }^{58}$ E. Rössler, K.-U. Hess, and V. Novikov, J. Non-Cryst. Solids 223, 207 (1998).

${ }^{59}$ I. Saika-Voivod, F. Sciortino, and P. H. Poole, Phys. Rev. E 69, 041503 (2004).

${ }^{60}$ J. Horbach, W. Kob, and K. Binder, Philos. Mag. B 77, 297 (1998).

${ }^{61}$ L. Berthier, Phys. Rev. E 76, 011507 (2007).

${ }^{62}$ M. Vogel and S. C. Glotzer, Phys. Rev. E 70, 061504 (2004).

${ }^{63}$ M. Vogel and S. C. Glotzer, Phys. Rev. Lett. 92, 255901 (2004).

${ }^{64}$ D. Coslovich and G. Pastore, J. Phys.: Condens. Matter 21, 285107 (2009).

${ }^{65}$ B. W. H. van Beest, G. J. Kramer, and R. A. van Santen, Phys. Rev. Lett. 64, 1955 (1990).

${ }^{66}$ C. J. Fennell and J. D. Gezelter, J. Chem. Phys. 124, 234104 (2006).

${ }^{67}$ P. P. Ewald, Ann. Phys. 369, 253 (1921).

${ }^{68}$ D. Wolf, Computer Simulation Studies in Condensed-Matter Physics VIII (Springer, 1995), pp. 57-68.

${ }^{69}$ L. Woodcock, J. Braunstein et al., Advances in Molten Salt Chemistry (Springer, Boston, MA, 1975).
${ }^{70}$ D. Wolf, P. Keblinski, S. R. Phillpot, and J. Eggebrecht, J. Chem. Phys. 110, 8254 (1999).

${ }^{71}$ A. Carré, L. Berthier, J. Horbach, S. Ispas, and W. Kob, J. Chem. Phys. 127, 114512 (2007).

${ }^{72}$ S. D. Stoddard and J. Ford, Phys. Rev. A 8, 1504 (1973).

${ }^{73}$ H. Reinisch and A. Heuer, Phys. Rev. Lett. 95, 155502 (2005).

${ }^{74}$ A. Saksaengwijit and A. Heuer, J. Phys.: Condens. Matter 19, 205143 (2007).

${ }^{75}$ D. Thirumalai, R. D. Mountain, and T. R. Kirkpatrick, Phys. Rev. A 39, 3563 (1989).

${ }^{76}$ T. F. Soules, G. H. Gilmer, M. J. Matthews, J. S. Stolken, and M. D. Feit, J. Non-Cryst. Solids 357, 1564 (2011).

${ }^{77}$ J. Mikkelsen, Jr., Appl. Phys. Lett. 45, 1187 (1984).

${ }^{78}$ G. Brebec, R. Seguin, C. Sella, J. Bevenot, and J. Martin, Acta Metall. 28, 327 (1980).

${ }^{79}$ J. Nocedal, Math. Comput. 35, 773 (1980).

${ }^{80}$ D. C. Liu and J. Nocedal, Math. Program. 45, 503 (1989).

${ }^{81}$ F. H. Stillinger and T. A. Weber, Phys. Rev. A 25, 978 (1982).

${ }^{82}$ J. Sarnthein, A. Pasquarello, and R. Car, Phys. Rev. B 52, 12690 (1995).

${ }^{83}$ K. Vollmayr-Lee and A. Zippelius, Phys. Rev. E 88, 052145 (2013).

${ }^{84}$ E. La Nave, H. E. Stanley, and F. Sciortino, Phys. Rev. Lett. 88, 035501 (2002).

${ }^{85}$ C. Donati, J. F. Douglas, W. Kob, S. J. Plimpton, P. H. Poole, and S. C. Glotzer, Phys. Rev. Lett. 80, 2338 (1998).

${ }^{86} \mathrm{~F}$. H. Stillinger and T. A. Weber, Science 225, 983 (1984).

${ }^{87}$ T. B. Schroder, S. Sastry, J. C. Dyre, and S. C. Glotzer, J. Chem. Phys. 112, 9834 (2000).

${ }^{88}$ D. J. Wales, Optim: A program for geometry optimisation and pathway calculations, http://www-wales.ch.cam.ac.uk/software.html.

${ }^{89}$ S. A. Trygubenko and D. J. Wales, J. Chem. Phys. 120, 2082 (2004).

${ }^{90}$ D. Sheppard, R. Terrell, and G. Henkelman, J. Chem. Phys. 128, 134106 (2008).

${ }^{91}$ G. Henkelman, B. P. Uberuaga, and H. Jónsson, J. Chem. Phys. 113, 9901 (2000).

${ }^{92}$ G. Henkelman and H. Jónsson, J. Chem. Phys. 113, 9978 (2000).

${ }^{93}$ L. J. Munro and D. J. Wales, Phys. Rev. B 59, 3969 (1999).

${ }^{94}$ J. M. Carr, S. A. Trygubenko, and D. J. Wales, J. Chem. Phys. 122, 234903 (2005).

${ }^{95}$ O. M. Becker and M. Karplus, J. Chem. Phys. 106, 1495 (1997).

${ }^{96}$ D. J. Wales, M. A. Miller, and T. R. Walsh, Nature 394, 758 (1998).

${ }^{97}$ D. J. Wales and J. P. K. Doye, Phys. Rev. B 63, 214204 (2001).

${ }^{98}$ J. P. K. Doye and D. J. Wales, J. Chem. Phys. 116, 3777 (2002).

${ }^{99}$ M. Griffiths, S. Niblett, and D. J. Wales, "Optimal alignment of structures for finite and period systems," J. Chem. Theory Comput. (published online 2017).

${ }^{100}$ P. G. Debenedetti and F. H. Stillinger, Nature 410, 259 (2001).

${ }^{101}$ V. K. de Souza, "Glassy dynamics and the potential energy landscape," Ph.D. thesis, University of Cambridge, 2008.

${ }^{102}$ V. K. de Souza, J. D. Stevenson, S. P. Niblett, J. D. Farrell, and D. J. Wales, J. Chem. Phys. 146, 124103 (2017).

${ }^{103}$ W. Kob, C. Donati, S. J. Plimpton, P. H. Poole, and S. C. Glotzer, Phys. Rev. Lett. 79, 2827 (1997). 